\title{
Extreme-Value Analysis of Self-Normalized Increments
}

\author{
Dissertation \\ zur Erlangung des mathematisch-naturwissenschaftlichen \\ Doktorgrades \\ "Doctor rerum naturalium" \\ der Georg-August-Universität Göttingen \\ vorgelegt von \\ Zakhar Kabluchko \\ aus Bryansk
}

Göttingen 2007 
D7

Referent: Prof. Dr. M. Denker

Korreferent: Prof. Dr. M. Schlather

Tag der mündlichen Prüfung: 23. April 2007 


\section{Acknowledgements.}

I would like to express my deep gratitude to my advisor, Prof. Manfred Denker, for his support, help and patience. I would like also to thank Prof. Axel Munk for numerous discussions on the topic of the dissertation and his enthusiasm. I'm also very grateful to Prof. Martin Schlather for being the Korreferent as well as to all people at the Institute of Mathematical Stochastics.

Finally, I would like to thank my parents. 



\section{Contents}

1 Introduction 1

1.1 Statement of Main Results . . . . . . . . . . . . . . . . . . . . 1

1.2 Related Results . . . . . . . . . . . . . . . . . . . . . . . . . 2

1.3 Method of the proof . . . . . . . . . . . . . 8

2 Extreme-Value Analysis of Self-Normalized Brownian Mo$\begin{array}{ll}\text { tion Increments } & 11\end{array}$

2.1 Introduction . . . . . . . . . . . . . . . . . . . 11

2.2 Locally Stationary Gaussian Fields . . . . . . . . . . . . . . . 12

2.3 Proof of the Main Theorem . . . . . . . . . . . . . . . . . . 18

3 Extreme-Value Analysis of Self-Normalized Totally Skewed $\alpha$-stable Increments 25

3.1 Introduction . . . . . . . . . . . . . . . . . . . . . . . . 25

3.2 Stable Lévy Processes . . . . . . . . . . . . . . . . . . . . . 27

3.3 Proof of Theorem 3.1.1|: Infinitesimal Maximal . . . . . . . . 29

3.4 The Maximum of a Drifted Totally Skewed Stable Process . . 36

3.5 Proof of Theorem 3.1.1|: Local Maximal . . . . . . . . . . . 39

3.6 Proof of Theorem 3.1.1|: Global Maximal ............ . 49

4 Almost sure limiting behavior of self-normalized increments 54

4.1 Introduction . . . . . . . . . . . . . . 54

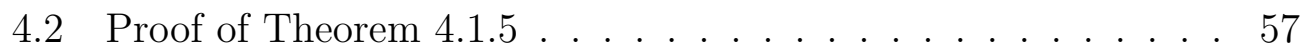





\section{Chapter 1}

\section{Introduction}

\subsection{Statement of Main Results}

Recall that a classical theorem of Lévy on the modulus of continuity of Brownian sample paths ([38], see e.g. [33, 40]) asserts that if $\{B(x), x \geq 0\}$ is the standard Brownian motion then, almost surely,

$$
\limsup _{n \rightarrow \infty} \frac{1}{\sqrt{2 \log n}} \sup _{\substack{x_{1}, x_{2} \in[0,1] \\ x_{2}-x_{1}=1 / n}} \frac{B\left(x_{2}\right)-B\left(x_{1}\right)}{\sqrt{x_{2}-x_{1}}}=1 .
$$

It is not difficult to deduce from this that

$$
\lim _{n \rightarrow \infty} \frac{1}{\sqrt{2 \log n}} \sup _{\substack{x_{1}, x_{2} \in[0,1] \\ x_{2}-x_{1} \geq 1 / n}} \frac{B\left(x_{2}\right)-B\left(x_{1}\right)}{\sqrt{x_{2}-x_{1}}}=1 .
$$

Our main result is the following theorem which can be considered as a distributional convergence version of Lévy's modulus of continuity.

Theorem 1.1.1. Let $\{B(x), x \geq 0\}$ be the standard Brownian motion. For $n>1$ set

$$
L_{n}=\sup _{\substack{x_{1}, x_{2} \in[0,1] \\ x_{2}-x_{1} \geq 1 / n}} \frac{B\left(x_{2}\right)-B\left(x_{1}\right)}{\sqrt{x_{2}-x_{1}}} .
$$

Then, for every $\tau \in \mathbb{R}$,

$$
\lim _{n \rightarrow \infty} \mathbf{P}\left[L_{n} \leq a_{n}+b_{n} \tau\right]=\exp \left(-e^{-\tau}\right),
$$


where the constants $a_{n}$ and $b_{n}$ are given by

$$
\begin{aligned}
& a_{n}=\sqrt{2 \log n}+\frac{3 / 2 \log _{2} n-\log 2 \sqrt{\pi}}{\sqrt{2 \log n}}, \\
& b_{n}=\frac{1}{\sqrt{2 \log n}} .
\end{aligned}
$$

Thus, the distribution of $L_{n}$, appropriately normalized, converges as $n \rightarrow \infty$ to the Gumbel distribution. We prove Theorem 1.1.1 in Chapter 2 .

In Chapter 3 we prove an analogue of Theorem 1.1.1 for totally skewed stable Lévy processes.

In Chapter 4 we prove a result on the almost sure limiting behavior of the multidimensional self-normalized increments. The methods of Chapter 4 are different from that of Chapters 2 and 3 .

We use the notation $\log _{2} n=\log \log n, \log _{3} n=\log \log \log n$, etc.

\section{$1.2 \quad$ Related Results}

The classical global law of the iterated logarithm for the Brownian motion says that if $\{B(x), x \geq 0\}$ is the standard Brownian motion then, almost surely,

$$
\limsup _{n \rightarrow \infty} \frac{B(n)}{\sqrt{2 n \log _{2} n}}=1,
$$

It is not difficult (see e.g. Theorem 14.15 in [50]) to deduce that

$$
\lim _{n \rightarrow \infty} \frac{1}{\sqrt{2 \log _{2} n}} \sup _{x \in[1, n]} \frac{B(x)}{\sqrt{x}}=1 .
$$

In 1956, Darling and Erdös [19] proved the following theorem which can be thought of as a distributional convergence version of the law of the iterated logarithm.

Theorem 1.2.1. Let $\{B(x), x>0\}$ be the standard Brownian motion. For $n>1$ define

$$
M_{n}=\sup _{x \in[1, n]} \frac{B(x)}{\sqrt{x}} .
$$

Then, for every $\tau \in \mathbb{R}$,

$$
\mathbf{P}\left[M_{n} \leq a_{n}+b_{n} \tau\right] \rightarrow \exp \left(-e^{-\tau}\right),
$$


where the constants $a_{n}$ and $b_{n}$ are given by

$$
\begin{aligned}
& a_{n}=\sqrt{2 \log _{2} n}+\frac{1 / 2 \log _{3} n-\log 2 \sqrt{\pi}}{\sqrt{2 \log _{2} n}}, \\
& b_{n}=\frac{1}{\sqrt{2 \log _{2} n}} .
\end{aligned}
$$

Of course, the same theorem holds also for

$$
M_{n}=\sup _{x \in[1 / n, 1]} \frac{B(x)}{\sqrt{x}},
$$

which gives a distributional convergence version of the local law of the iterated logarithm.

The classical Hartman-Wintner law of the iterated logarithm says that if $\left\{X_{i}, i \in \mathbb{N}\right\}$ is an i.i.d. sequence of random variables satisfying $\mathbf{E}\left[X_{i}\right]=0$, $\mathbf{E}\left[X_{i}^{2}\right]=1$ then

$$
\limsup _{n \rightarrow \infty} \frac{S_{n}}{\sqrt{2 n \log _{2} n}}=1,
$$

where $S_{n}=X_{1}+\ldots+X_{n}$. Using this, it is not difficult to show (see Theorem 14.15 in [50]) that

$$
\lim _{n \rightarrow \infty} \frac{1}{\sqrt{2 \log _{2} n}} \sup _{k \in\{1, \ldots, n\}} \frac{S_{k}}{\sqrt{k}}=1 .
$$

Using Theorem 1.2.1 and a strong approximation argument, Darling and Erdös deduced the following distributional convergence version of the law of the iterated logarithm for i.i.d. sequences.

Theorem 1.2.2 (Darling-Erdös). Let $\left\{X_{i}, i \in \mathbb{N}\right\}$ be i.i.d. random variables subject to $\mathbf{E}\left[X_{i}\right]=0, \mathbf{E}\left[X_{i}^{2}\right]=1$ and $\mathbf{E}\left[\left|X_{i}\right|^{3}\right]<\infty$. Define $S_{n}=$ $X_{1}+\ldots+X_{n}$ and

$$
M_{n}=\max _{k \in\{1, \ldots, n\}} \frac{S_{k}}{\sqrt{k}} .
$$

Then, for every $\tau \in \mathbb{R}$,

$$
\mathbf{P}\left[M_{n} \leq a_{n}+b_{n} \tau\right] \rightarrow \exp \left(-e^{-\tau}\right),
$$


where the constants $a_{n}$ and $b_{n}$ are given by

$$
\begin{aligned}
& a_{n}=\sqrt{2 \log _{2} n}+\frac{1 / 2 \log _{3} n-\log 2 \sqrt{\pi}}{\sqrt{2 \log _{2} n}}, \\
& b_{n}=\frac{1}{\sqrt{2 \log _{2} n}} .
\end{aligned}
$$

The moment condition in the above theorem can be relaxed. Indeed, Einmahl [22] has found the following necessary and sufficient condition for the Darling-Erdös theorem to hold.

Theorem 1.2.3. Let $\left\{X_{i}, i \in \mathbb{N}\right\}$ be i.i.d. random variables with $\mathbf{E}\left[X_{i}\right]=0$, $\mathbf{E}\left[X_{i}^{2}\right]=1$. Define $M_{n}$ like in Theorem 1.2.2. Then the following statements are equivalent:

1. $\mathbf{P}\left[M_{n} \leq a_{n}+b_{n} \tau\right] \rightarrow \exp \left(-e^{-\tau}\right)$.

2. $\mathbf{E}\left[X_{i}^{2} 1_{\left|X_{i}>t\right|}\right]=o(1 / \log \log t)$ as $t \rightarrow \infty$.

Both the law of the iterated logarithm and its distributional convergence version were generalized in different directions. See [23] for a generalization of the Darling-Erdös theorem to martingale differences and [30] for a version for long-range dependent variables. For an almost-sure version of the DarlingErdös theorem see [7, 9, 10].

Let us also mention an alternative, different from the Darling-Erdös theorem, distributional version of the law of the iterated logarithm, proved in [34].

Theorem 1.2.4. Let $\{B(x), x \geq 0\}$ be the standard Brownian motion. Define

$$
M_{n}=\sup _{x \geq n} \frac{B(x)}{\sqrt{2 x \log _{2} x}} .
$$

Then, for every $\tau \in \mathbb{R}$,

$$
\lim _{n \rightarrow \infty} \mathbf{P}\left[M_{n} \leq a_{n}+b_{n} \tau\right]=\exp \left(-e^{-\tau}\right),
$$

where the constants $a_{n}$ and $b_{n}$ are given by

$$
\begin{aligned}
& a_{n}=1+\frac{3 / 2 \log _{3} n-\log _{4} n-\log (3 / \sqrt{2})}{2 \log _{2} n}, \\
& b_{n}=\frac{1}{2 \log _{2} n} .
\end{aligned}
$$


Another classical theorem concerning the almost sure sample path behavior of the Brownian motion is Lévy's modulus of continuity. It says that if $\{B(x), x \geq 0\}$ is the standard Brownian motion then, almost surely,

$$
\limsup _{n \rightarrow \infty} \frac{1}{\sqrt{2 \log n}} \sup _{\substack{x_{1}, x_{2} \in[0,1] \\ x_{2}-x_{1}=1 / n}} \frac{B\left(x_{2}\right)-B\left(x_{1}\right)}{\sqrt{x_{2}-x_{1}}}=1 .
$$

Note that although Lévy's modulus of continuity is usually stated in the form given above (see e.g. [33, 40]), it is not difficult to show that (1.3) remains true with lim instead of limsup.

If we set, for $n>1$,

$$
L_{n}=\sup _{\substack{x_{1}, x_{2} \in[0,1] \\ x_{2}-x_{1} \geq 1 / n}} \frac{B\left(x_{2}\right)-B\left(x_{1}\right)}{\sqrt{x_{2}-x_{1}}}
$$

then

$$
\lim _{n \rightarrow \infty} \frac{1}{\sqrt{2 \log n}} L_{n}=1
$$

Our main result, Theorem 1.1.1, asserts that, appropriately normalized, the distribution of $L_{n}$ converges weakly to the Gumbel distribution. We shall prove Theorem 1.1.1 in Chapter 2. While the proofs of both Theorem 1.2.1 and Theorem 1.2.4 rely on explicit calculations with the Ornstein-Uhlenbeck process, our proof of Theorem 1.1.1 uses Pickands' method of double sums. A quantity similar to $L_{n}$ was considered in [20] where the following result was proved

Theorem 1.2.1. Let $\{B(x), x \geq 0\}$ be the standard Brownian motion. Then

$$
\sup _{\substack{x_{1}, x_{2} \in[0,1] \\ x_{2}>x_{1}}} \frac{B\left(x_{2}\right)-B\left(x_{1}\right)}{\sqrt{x_{2}-x_{1}}}-\sqrt{2 \log \frac{1}{x_{2}-x_{1}}}
$$

is finite almost surely.

Note, however, that this result follows from the Erdös-Chung-Sirao integral test (see [17] or [33, §1.9]).

The following theorem proved in [20, 21] is a discrete version of Theorem 1.2.1. 
Theorem 1.2.5. Let $\left\{X_{i}, i \in \mathbb{N}\right\}$ be an i.i.d. sequence of standard normal variables. Define $S_{n}=X_{1}+\ldots+X_{n}$ and

$$
L_{n}=\max _{1 \leq i<j \leq n}\left(\frac{S_{j}-S_{i}}{\sqrt{j-i}}-\sqrt{2 \log \frac{n}{j-i}}\right) .
$$

Then the distribution of $L_{n}$ converges weakly as $n \rightarrow \infty$.

Unfortunately, we are unable to prove the following discrete counterpart of Theorem 1.1.1 even in the case of normally distributed variables.

Statement 1.2.6. Let $\left\{X_{i}, i \in \mathbb{N}\right\}$ be an i.i.d. sequence of random variables subject to $\mathbf{E}\left[X_{i}\right]=0, \mathbf{E}\left[X_{i}^{2}\right]=1$ and some additional moment condition. Define $S_{n}=X_{1}+\ldots+X_{n}$ and

$$
L_{n}=\max _{1 \leq i<j \leq n} \frac{S_{j}-S_{i}}{\sqrt{j-i}} .
$$

Then the distribution of $L_{n}$, appropriately normalized, converges to the Gumbel distribution.

The almost sure limiting behavior of $L_{n}$ from the above statement was studied in [50, §14.3] and [52, 53]. In particular, Shao [52] proves the following result.

Theorem 1.2.7. Let $\left\{X_{i}: i \in \mathbb{N}\right\}$ be i.i.d. random variables. Suppose that $\mathbf{E}\left[X_{i}\right]=0, \mathbf{E}\left[X_{i}^{2}\right]=1$ and that $\varphi(t)=\mathbf{E}\left[e^{t X_{1}}\right]$ exists finitely in some interval containing 0 . Set $S_{n}=X_{1}+\ldots+X_{n}$ and

$$
L_{n}=\max _{1 \leq i<j \leq n} \frac{S_{j}-S_{i}}{\sqrt{j-i}} .
$$

Then, almost surely,

$$
\lim _{n \rightarrow \infty} \frac{1}{\sqrt{2 \log n}} L_{n}=\alpha^{*},
$$

where $\alpha^{*} \in[1, \infty]$ is a constant depending on the distribution of $X_{i}$.

It follows from the above theorem that the normalizing constants in Statement 1.2.6 have to depend on the distribution of $X_{i}$. In particular, it is impossible to deduce Statement 1.2 .6 from Theorem 1.1.1 by any straightforward strong approximation argument. 
Both the law of the iterated logarithm and Lévy's modulus of continuity were generalized to $\alpha$-stable Lévy processes. We consider here only the more difficult case of totally skewed processes.

The following theorem [26, 41] is the law of the iterated logarithm for totally skewed $\alpha$-stable processes, see [41] for more information.

Theorem 1.2.8. Let $\{\xi(x), x \geq 0\}$ be an $\alpha$-stable Lévy process with $\alpha \in(1,2]$ and skewness parameter $\beta=-1$. Then

$$
\limsup _{n \rightarrow \infty} \frac{\xi(n)}{n^{1 / \alpha}\left(\log _{2} n\right)^{(\alpha-1) / \alpha}}=B_{\alpha}^{-(\alpha-1) / \alpha},
$$

where

$$
B_{\alpha}=(\alpha-1) \alpha^{-\alpha /(\alpha-1)}|\cos (\pi \alpha / 2)|^{1 /(\alpha-1)} .
$$

There are also analogous results for $\alpha \in(0,1)$ and $\alpha=1$ (see [41]).

The following distributional convergence version of the law of the iterated logarithm for totally skewed $\alpha$-stable processes was proved by Bertoin in [12].

Theorem 1.2.9. Let $\{\xi(x), x \geq 0\}$ be an $\alpha$-stable Lévy process with $\alpha \in(1,2]$ and skewness parameter $\beta=-1$. For $n>1$ set

$$
M_{n}=\sup _{x \in[1, n]} \frac{\xi(x)}{x^{1 / \alpha}} \text {. }
$$

Then, for every $\tau \in \mathbb{R}$,

$$
\lim _{n \rightarrow \infty} \mathbf{P}\left[M_{n} \leq a_{n}+b_{n} \tau\right]=\exp \left(-e^{-\tau}\right),
$$

where the constants $a_{n}$ and $b_{n}$ are given by

$$
\begin{aligned}
a_{n} & =\frac{1}{B_{\alpha}^{(\alpha-1) / \alpha}}\left(\left(\log _{2} n\right)^{(\alpha-1) / \alpha}+\frac{\alpha-1}{\alpha} \frac{1 / 2 \log _{3} n-\log \sqrt{2 \alpha \pi}}{\left(\log _{2} n\right)^{1 / \alpha}}\right), \\
b_{n} & =\frac{1}{B_{\alpha}^{(\alpha-1) / \alpha}} \frac{\alpha-1}{\alpha} \frac{1}{\left(\log _{2} n\right)^{1 / \alpha}} .
\end{aligned}
$$

Here, $B_{\alpha}$ is the same constant as in the previous theorem.

There is also a version for $\alpha \in(0,1)$, see [12].

The next theorem [29, 41] ist the analogue of Lévy's modulus of continuity for totally-skewed $\alpha$-stable processes. 
Theorem 1.2.10. Let $\{\xi(x), x \geq 0\}$ be an $\alpha$-stable Lévy process with $\alpha \in$ $(1,2]$ and skewness parameter $\beta=-1$. For $n>1$ set

$$
L_{n}=\sup _{\substack{x_{1}, x_{2} \in[0,1] \\ x_{2}-x_{1} \geq 1 / n}} \frac{\xi\left(x_{2}\right)-\xi\left(x_{1}\right)}{\left(x_{2}-x_{1}\right)^{1 / \alpha}} .
$$

Then

$$
\lim _{n \rightarrow \infty} \frac{L_{n}}{(\log n)^{(\alpha-1) / \alpha}}=B_{\alpha}^{-(\alpha-1) / \alpha},
$$

where $B_{\alpha}$ is the same as in Theorem 1.2.8.

The main result of Chapter 3, Theorem 3.1.1, says that, appropriately normalized, the distribution of $L_{n}$ converges to the Gumbel distribution.

\subsection{Method of the proof}

A natural method to use for the proof of Theorem 1.1.1 is the double sum method. The double sum method was introduced by Pickands in [43, 44], where the following theorem was proved

Theorem 1.3.1 (Pickands). Let $\{X(t), t \in \mathbb{R}\}$ be a stationary centered and normed gaussian process with continuous sample paths. Suppose that the covariance function $r(t)=\mathbf{E}[X(0) X(t)]$ satisfies the following conditions:

1. $r(t)=1-C|t|^{\alpha}+o\left(|t|^{\alpha}\right)$ as $t \rightarrow 0$ for some $\alpha \in(0,2]$.

2. $r(t) \log t \rightarrow 0$ as $t \rightarrow \infty$.

For $n>0$ define

$$
M_{n}=\sup _{t \in[0, n]} X(t)
$$

Then, for every $\tau \in \mathbb{R}$,

$$
\lim _{n \rightarrow \infty} \mathbf{P}\left[M_{n} \leq a_{n}+b_{n} \tau\right]=\exp \left(-e^{-\tau}\right),
$$

where the constants $a_{n}$ and $b_{n}$ are given by

$$
\begin{aligned}
& a_{n}=\sqrt{2 \log n}+\frac{\frac{2-\alpha}{2 \alpha} \log _{2} n+\log \left(H_{\alpha} C(2 \pi)^{-1 / 2} 2^{(2-\alpha) / 2 \alpha}\right)}{\sqrt{2 \log n}}, \\
& b_{n}=\frac{1}{\sqrt{2 \log n}} .
\end{aligned}
$$

Here, $H_{\alpha} \in(0, \infty)$ is some constant. 
We refer to [36, Chapter 12] and [46] for two descriptions of Pickands' method as well as for simplifications in the original proof of Theorem 1.3.1. See also [48, 49, 14, 3] for generalizations of Theorem 1.3.1.

Here, we recall only the basic idea of Pickands' method. It consists of three stages. Let $\left\{X(t), t \in \mathbb{R}^{d}\right\}$ be centered and normed gaussian field. Let $\left\{G_{n}, n>0\right\}, G_{n} \subset \mathbb{R}^{d}$ be a growing family of sets. The goal is to prove that the distribution of $M_{n}=\sup _{t \in G_{n}} X(t)$ converges, after rescaling, to the Gumbel distribution.

At the first stage (infinitesimal maxima), one obtains an estimate of the form

$$
\mathbf{P}\left[\sup _{t \in Q\left(t_{0}, u\right)} X(t)>u\right] \sim C \mathbf{P}\left[X\left(t_{0}\right)>u\right], \quad u \rightarrow \infty .
$$

Here, $t_{0} \in \mathbb{R}^{d}$ and $Q\left(t_{0}, u\right) \ni t_{0}$ is a small set depending on $u$ and converging to $t_{0}$ as $u \rightarrow \infty$. $C$ is a constant depending on the precise structure of the set $Q\left(t_{0}, u\right)$.

At the second stage (local maxima), one investigates the asymptotic behavior of $\mathbf{P}\left[\sup _{x \in K} X(t)>u\right]$ for a compact set $K$ of finite positive measure. To this end, one divides the set $K$ into small sets of the form $Q\left(t_{i}, u\right), i \in I$, considered at the first stage and proves that the events $\sup _{t \in Q\left(t_{i}, u\right)} X(t)>u$ are getting independent as $u \rightarrow \infty$. Using the Bonferroni inequality one obtains

$$
\mathbf{P}\left[\sup _{x \in K} X(t)>u\right] \approx \sum_{i} \mathbf{P}\left[\sup _{t \in Q\left(t_{i}, u\right)} X(t)>u\right] \approx C u^{D} \mathbf{P}[X(0)>u]
$$

for some constant $D$ depending on the structure of the field $X$.

At the third stage (global maxima), one considers $\mathbf{P}\left[\sup _{t \in G_{n}} X(t)>u_{n}\right]$ for a growing family $G_{n}$ of sets and a sequence $u_{n}$ converging to $\infty$ as $n \rightarrow \infty$. One divides $G_{n}$ into $\approx n$ approximately equal peaces $K_{i}$, of the form considered at the second stage, and proves that the events $\sup _{t \in K_{i}} X(t)>u_{n}$ are getting independent as $n \rightarrow \infty$. The peaces $K_{i}$ and the sequence $u_{n}$ are chosen in such a way that $\mathbf{P}\left[\sup _{t \in K_{i}} X(t)>u_{n}\right] \sim e^{-\tau} / n, n \rightarrow \infty$. Since the maxima over the different $K_{i}$ are approximately independent, one obtains by the Poisson limit theorem the desired result

$$
\mathbf{P}\left[\sup _{t \in G_{n}} X(t)>u_{n}\right] \rightarrow \exp \left(-e^{-\tau}\right), \quad n \rightarrow \infty .
$$


Originally, Pickands' method was used for stationary processes defined on $\mathbb{R}$. Later, it was extended to stationary processes on $\mathbb{R}^{d}[14$, to stationary nongaussian processes [1], etc. A general class of gaussian fields, called locally stationary fields, to which the first two stages of Pickands' method can be applied was introduced in [42]. However, both the definition of this class and the statement of the main result in 42 seem to contain errors. A correct version is given in [16], see also [31] for the one-dimensional case. We recall the definition of locally stationary gaussian fields in Section 2.2 and show that the field of self-normalized Brownian motion increments defined by

$$
X(x, y)=\frac{B(x+y)-B(x)}{y^{1 / 2}}, \quad y>0,
$$

belongs to this class. Here, $\{B(t), t>0\}$ is the standard Brownian motion. The third stage of Pickands' method is done in Section 2.3.

Let us say a few words about the extension of Theorem 1.1.1 to the totally skewed $\alpha$-stable case. This is done in Chapter 3, see Theorem 3.1.1 for the statement of the main result. First note that we consider only totally skewed to the left processes. For, otherwise, the process has positive jumps, and it is not difficult to prove that the functional $L_{n}$ from Theorem 3.1.1 converges, after normalization, to the length of the largest jump of the process. Since it is known that the largest jump has Frechet distribution, the analogue of Theorem 3.1.1 in the non-totally-skewed case becomes rather trivial. Thus, the only interesting cases are $\alpha \in(1,2], \beta=-1 ; \alpha=1, \beta=-1$ and $\alpha \in$ $(0,1), \beta=-1$. In Theorem 3.1.1, only the first case is considered. The other cases can be treated by the same methods.

The main technical difficulty of Pickands' method is proving the approximate independence of events of the form $\sup _{t \in Q\left(t_{i}, u\right)} X(t)>u$ (second stage) and $\sup _{t \in K_{i}}>u_{n}$ (third stage). If the process is gaussian, such tools as Fernique Inequality, Borell Inequality, Slepian's Lemma, Berman's Inequality, etc. are usually used. Since all these tools are not available in the $\alpha$-stable setting, we make use instead of the concrete structure of the process $X$. Our methods are similar to that of Albin [2, 3, 4, 5], who studied the extreme-value behavior of $\alpha$-stable (and, more generally, infinitely divisible) Ornstein-Uhlenbeck processes. Some results of Albin, in particular that from [2] and, partially, [4] can be obtained by doing explicit calculations with Ornstein-Uhlenbeck processes like in [12] without using Pickands' method. However, in the case of self-normalized increments, explicit calculations seem impossible. 


\section{Chapter 2}

\section{Extreme-Value Analysis of Self-Normalized Brownian Motion Increments}

\section{$2.1 \quad$ Introduction}

In this chapter we prove Theorem 1.1.1 from Chapter 1. Let us describe briefly the method of the proof and fix some notation.

Let

$$
\mathbb{H}=\left\{t=(x, y) \in \mathbb{R}^{2} \mid y>0\right\}
$$

denote the open upper half-plane. A point $t=(x, y) \in \mathbb{H}$ will be often identified with the interval $[x, x+y] \subset \mathbb{R}$. There is a natural action of the group of affine transformations of the real line on $\mathbb{H}$ defined as follows. If $g: x \mapsto a x+b$, where $a>0, b \in \mathbb{R}$, is an affine transformation of $\mathbb{R}$, then the action of $g$ on $\mathbb{H}$ is given by

$$
g(t)=(a x+b, a y), \quad t=(x, y) \in \mathbb{H} .
$$

Let $\{B(x), x \geq 0\}$ be the standard Brownian motion. Define the random field $\{X(t), t=(x, y) \in \mathbb{H}\}$ of self-normalized Brownian increments by

$$
X(t)=\frac{B(x+y)-B(x)}{y^{1 / 2}} .
$$

Note that the field $X$ is gaussian. Note also that for each $t \in \mathbb{H}$ the distribution of $X(t)$ is standard normal. 
The following invariance property of the field $X$ will be useful

Proposition 2.1.1. Let $g$ be an affine transformation of $\mathbb{R}$. Then, for each $t_{1}, \ldots, t_{n} \in \mathbb{H}$, the joint distribution of $X\left(g\left(t_{1}\right)\right), \ldots, X\left(g\left(t_{n}\right)\right)$ coincides with the joint distribution of $X\left(t_{1}\right), \ldots, X\left(t_{n}\right)$.

Proof. Suppose first that $g(x)=x+b$. Then the statement of the proposition follows from the stationarity of the increments of the Brownian motion. Suppose now that $g(x)=a x$. Then the proposition follows from the scaling property of the Brownian motion. Since any affine transformation of $\mathbb{R}$ is a composition of $x \mapsto a x$ and $x \mapsto x+b$, the proposition follows.

The above proposition allows us to state Theorem 1.1.1 in the following, equivalent form.

Theorem 2.1.2. For $n>1$ let $H(n)$ be the triangle $\{(x, y) \in \mathbb{H} \mid x \in$ $[0, n], y \in[1, n-x]\}$. Define the random field $X$ by (2.1). Then, for each $\tau \in \mathbb{R}$,

$$
\lim _{n \rightarrow \infty} \mathbf{P}\left[\sup _{t \in H(n)} X(t) \leq a_{n}+b_{n} \tau\right]=\exp \left(-e^{-\tau}\right),
$$

where $a_{n}, b_{n}$ are constants defined by (1.1) and (1.2).

The rest of the chapter is devoted to the proof of Theorem 2.1.2.

In Section 2.2, we recall the notion of locally stationary gaussian field introduced in [6, 31, 42, 16], give some examples and show that the field $X$ belongs to this class. The main result of this section is Corollary 2.2.12.

The proof of Theorem 2.1.2 is given in Section 2.3.

\subsection{Locally Stationary Gaussian Fields}

Given a centered normed gaussian field $\left\{X(t), t \in \mathbb{R}^{n}\right\}$ we would like to obtain an exact asymptotics of the high excursion probability of $X$ over a given compact set $K$, i.e. a result of the form

$$
\mathbf{P}\left[\sup _{t \in K} X(t)>u\right] \sim C_{K} u^{D} e^{-u^{2} / 2}, \quad u \rightarrow \infty
$$

for a number $D$ depending on the structure of the field and a constant $C_{K}$ depending on the set $K \subset \mathbb{R}^{n}$ and the structure of the field. 
In this section, we define a class of gaussian fields for which this is possible. These so-called locally stationary fields were introduced in [6, 31, 42, 16].

First we recall the following

Definition 2.2.1. A function $f: \mathbb{R}^{d} \rightarrow \mathbb{R}$ is called homogeneous of order $\alpha>0$ if for each $s \in \mathbb{R}^{d}$ and $\lambda \in \mathbb{R}$

$$
f(\lambda s)=|\lambda|^{\alpha} f(s) .
$$

In particular, homogeneous functions are symmetric, i.e. they satisfy $f(s)=$ $f(-s)$. Let $H(\alpha)$ be the set of all continuous homogeneous functions of order $\alpha$. For $f \in H(\alpha)$ define $\|f\|=\sup _{\|t\|_{2}=1} f(t)$. With this norm, $H(\alpha)$ is a Banach space which can be identified with the space $C\left(\mathbb{S}^{d-1}\right)$ of continuous functions on the unit sphere in $\mathbb{R}^{d}$.

Let $H^{+}(\alpha)$ be the cone of all strictly positive functions in $H(\alpha)$.

Now we are ready to define the notion of locally stationary gaussian field.

Definition 2.2.2 (see [16]). Let $\{X(t), t \in D\}$ be a centered and normed gaussian field defined on some domain $D \subset \mathbb{R}^{d}$. Let $r\left(t_{1}, t_{2}\right)=\mathbf{E}\left[X\left(t_{1}\right) X\left(t_{2}\right)\right]$ be the correlation function of $X$. The field $X$ is called locally stationary if a number $\alpha \in(0,2]$ and a continuous map $C_{\bullet}: D \rightarrow H^{+}(\alpha)$ exist such that

$$
\lim _{\|s\|_{2} \rightarrow 0} \frac{1-r(t, t+s)}{C_{t}(s)}=1
$$

uniformly on compacts in $t \in D$. The number $\alpha$ and the function $C$. : $D \rightarrow H(\alpha)$ are referred to as the index and the local structure of the field $X$ respectively.

The next proposition gives a representation for the local structure of a locally stationary field. Note that the corresponding representation in [42] seems to be false.

Proposition 2.2.3. Let $\{X(t), t \in D\}$ be a locally stationary gaussian field of index $\alpha$ with local structure $C_{t}(s)$. Then, for each fixed $t \in D$, the function $C_{t}(\cdot)$ is negative definite. Moreover, there is a finite measure $\Gamma_{t}$ on $\mathbb{S}^{d-1}$ such that the following representation holds

$$
C_{t}(s)=\int_{\mathbb{S}^{d-1}}|(s, x)|^{\alpha} d \Gamma_{t}(x) .
$$

The support of $\Gamma_{t}$ is not contained in any proper linear subspace of $\mathbb{R}^{d}$. 
Proof. Recall (see e.g. [8]) that a continuous function $f: \mathbb{R}^{d} \rightarrow \mathbb{R}$ satisfying $f(s)=f(-s)$ and $f(0)=0$ is called negative definite if for each $s_{1}, \ldots, s_{n} \in$ $\mathbb{R}^{d}$ the matrix

$$
\left(f\left(s_{i}\right)+f\left(s_{j}\right)-f\left(s_{i}-s_{j}\right)\right)_{i, j=1, \ldots, n}
$$

is positive definite. For $u>0$ set $q=q(u)=u^{-2 / \alpha}$. Define $Y_{i}=Y_{i}(u)$ by

$$
Y_{i}=u\left(X\left(t+q s_{i}\right)-u\right) .
$$

Consider the joint distribution of $\left\{Y_{i}, i=1, \ldots, n\right\}$ conditioned on $X(t)=u$. It is (non-centered) gaussian and the well-known formulas show that the covariance matrix is

$$
\left(u^{2} r\left(t+q s_{i}, t+q s_{j}\right)-u^{2} r\left(t, t+q s_{i}\right) r\left(t, t+q s_{j}\right)\right)_{i, j=1, \ldots, n} .
$$

It follows from the definition of local stationarity that, as $u \rightarrow \infty$, this converges to

$$
\left(C_{t}\left(s_{i}\right)+C_{t}\left(s_{j}\right)-C_{t}\left(s_{i}-s_{j}\right)\right)_{i, j=1, \ldots, n} .
$$

Since the limit of positive definite matrices is positive definite, it follows that $C_{t}(\cdot)$ is a negative definite function.

By Schoenbergs theorem (see e.g. [8, Theorem 2.2]) the function $\exp \left(-C_{t}(\cdot)\right)$ is positive definite and thus is the characteristic function of some symmetric probability measure $\mu$ on $\mathbb{R}^{n}$. Since $C_{t}(\cdot)$ is homogeneous of order $\alpha$, the measure $\mu$ is stable of order $\alpha$. The remaining part of the proposition follows from the classification of symmetric stable measures on $\mathbb{R}^{n}$ (see e.g. [51]).

Now we give some examples of locally stationary fields.

Example 2.2.4 (see [43]). Let $\{X(t), t \in \mathbb{R}\}$ be centered and normed stationary gaussian process with correlation function $r(t)=\mathbf{E}[X(0) X(t)]$. Suppose that

$$
r(t)=1-C|t|^{\alpha}+o\left(\left|t^{\alpha}\right|\right)
$$

for some $C>0$ and $\alpha \in(0,2]$. Then $X$ is locally stationary of index $\alpha$. The local structure is given by $C_{t}(s)=C$. Examples include, to mention only a few, $r(t)=\exp \left(-|t|^{\alpha}\right)$ (the generalized Ornstein-Uhlenbeck process), $r(t)=\left(1+|t|^{\alpha}\right)^{-\beta}$ for $\alpha \in(0,2]$ and $\beta>0$ (the generalized Cauchy model, see [27]). 
Example 2.2.5 (see [6]). Let $\{B(t), t \geq 0\}$ be the standard Brownian motion. The self-normalized Brownian motion is the process $\{X(t), t>0\}$ defined by

$$
X(t)=B(t) / \sqrt{t} .
$$

The self-normalized Brownian motion is locally stationary with index $\alpha=1$. The local structure is given by $C_{t}(s)=\frac{|s|}{2 t}$.

Proof. Using that $\operatorname{Cov}\left(B\left(t_{1}\right), B\left(t_{2}\right)\right)=\min \left(t_{1}, t_{2}\right)$ we obtain, for $s>0$,

$$
r(t, t+s)=\operatorname{Cov}(X(t), X(t+s))=\frac{t}{\sqrt{t(t+s)}}=1-\frac{s}{2 t}+O\left(s^{2}\right) .
$$

For $s<0$ we obtain

$$
r(t, t+s)=\operatorname{Cov}(X(t), X(t+s))=\frac{t+s}{\sqrt{t(t+s)}}=1+\frac{s}{2 t}+O\left(s^{2}\right) .
$$

Example 2.2.6 (see [6, 16]). Let $\{B(x), x>0\}$ be the standard Brownian motion. Then the process $\{X(t), t=(x, y) \in \mathbb{H}\}$ of self-normalized Brownian increments defined in Section 2.1 by

$$
X(t)=\frac{B(x+y)-B(x)}{\sqrt{y}}
$$

is locally stationary with index $\alpha=1$. The local structure is given by

$$
C_{t}(s)=\left(\left|s_{x}\right|+\left|s_{x}+s_{y}\right|\right) /(2 y),
$$

where $t=(x, y) \in \mathbb{H}$ and $s=\left(s_{x}, s_{y}\right) \in \mathbb{R}^{2}$.

Proof. Let $t=(x, y) \in \mathbb{H}$ and $s=\left(s_{x}, s_{y}\right) \in \mathbb{R}^{2}$. Suppose that $s_{x}>0$, $s_{x}+s_{y}>0$. Then

$$
\begin{aligned}
r(t, s)= & \operatorname{Cov}(X(t), X(t+s))= \\
& \frac{1}{\sqrt{y\left(y+s_{y}\right)}} \operatorname{Cov}\left(B(x+y)-B(x), B\left(x+y+s_{x}+s_{y}\right)-B\left(x+s_{x}\right)\right)= \\
& \frac{y-s_{x}}{\sqrt{y\left(y+s_{y}\right)}}=1-\frac{s_{x}}{y}-\frac{s_{y}}{2 y}+o\left(s_{x}, s_{y}\right)= \\
& 1-\left(\left|s_{x}\right|+\left|s_{x}+s_{y}\right|\right) /(2 y)+o\left(s_{x}, s_{y}\right) .
\end{aligned}
$$


Suppose that $s_{x}>0, s_{x}+s_{y}<0$. Then

$$
\begin{aligned}
r(t, s)= & \operatorname{Cov}(X(t), X(t+s))= \\
& \frac{1}{\sqrt{y\left(y+s_{y}\right)}} \operatorname{Cov}\left(B(x+y)-B(x), B\left(x+y+s_{x}+s_{y}\right)-B\left(x+s_{x}\right)\right)= \\
& \frac{y+s_{y}}{\sqrt{y\left(y+s_{y}\right)}}=1+\frac{s_{y}}{2 y}+o\left(s_{x}, s_{y}\right)= \\
& 1-\left(\left|s_{x}\right|+\left|s_{x}+s_{y}\right|\right) /(2 y)+o\left(s_{x}, s_{y}\right) .
\end{aligned}
$$

Other cases can be treated analogously.

Example 2.2.7. Let $\{B(t), t \in[0,1]\}$ be the Brownian bridge. Then the self-normalized Brownian bridge $\{X(t), t \in(0,1)\}$ defined by

$$
X(t)=B(t) / \sqrt{t(1-t)}
$$

is locally stationary with index $\alpha=1$ and local structure $C_{t}(s)=\frac{|s|}{2 t}+\frac{|s|}{2(1-t)}$. The next example is a multidimensional generalization of Example 2.2.6.

Example 2.2.8. Let $\{\xi(A), A \in \mathcal{B}\}$ be the white noise on $\left([0,1]^{d}, \mathcal{B}\right.$, Leb). This means that we are given a centered gaussian process $\xi$ indexed by the collection $\mathcal{B}$ of all Borel subsets of the unit cube $[0,1]^{d}$ such that

$$
\operatorname{Cov}\left(\xi\left(A_{1}\right), \xi\left(A_{2}\right)\right)=\operatorname{Leb}\left(A_{1} \cap A_{2}\right) \quad \text { for each } A_{1}, A_{2} \in \mathcal{B},
$$

where Leb denotes the Lebesgue measure. A set of the form

$$
\left[x_{1}, y_{1}\right] \times \ldots \times\left[x_{d}, y_{d}\right], \quad 0 \leq x_{i}<y_{i} \leq 1
$$

is called rectangle. Let

$$
\mathcal{R}=\left\{\left(x_{1}, y_{1}, \ldots, x_{d}, y_{d}\right) \in \mathbb{R}^{2 d} \mid 0 \leq x_{i}<y_{i} \leq 1\right\}
$$

be the collection of all rectangles. Define a process $\{X(R), R \in \mathcal{R}\}$ indexed by rectangles by

$$
X(R)=\xi(R) / \sqrt{\operatorname{Leb}(\mathrm{R})} .
$$

Then $X$ is locally stationary on $\mathcal{R}$ of index $\alpha=1$. The local structure is given by

$$
C_{t}(s)=\sum_{i=1}^{d}\left(\left|s_{i x}\right|+\left|s_{i x}+s_{i y}\right|\right) /\left(2 t_{i y}\right)
$$

where

$$
t=\left(t_{1 x}, t_{1 y}, \ldots, t_{d x}, t_{d y}\right) \in \mathcal{R}, \quad s=\left(s_{1 x}, s_{1 y}, \ldots, s_{d x}, s_{d y}\right) \in \mathbb{R}^{2 d} .
$$


Example 2.2.9. The Brownian motion with multidimensional time, introduced by Lévy, is a centered gaussian process $\left\{B(t), t \in \mathbb{R}^{d}\right\}$ with the covariance function

$$
\operatorname{Cov}(B(t), B(s))=\frac{1}{2}\left(\|t\|_{2}+\|s\|_{2}-\|t-s\|_{2}\right),
$$

where $\|t\|_{2}$ denotes the euclidian norm of $t$. Then the process $\{X(t), t \in$ $\left.\mathbb{R}^{d} \backslash\{0\}\right\}$ defined by

$$
X(t)=B(t) / \sqrt{\|t\|_{2}}
$$

is locally stationary of index $\alpha=1$. The local structure is given by

$$
C_{t}(s)=\frac{\|s\|_{2}}{2\|t\|_{2}}, \quad t \in \mathbb{R}^{d} \backslash\{0\}, s \in \mathbb{R}^{d} .
$$

Further examples of locally stationary gaussian fields include e.g. the selfnormalized Brownian sheet, the self-normalized Brownian pillow, the selfnormalized fractional Brownian motion etc.

The next proposition (see [42, 16]) corresponds to the first stage of Pickands' method.

Proposition 2.2.10. Let $\{X(t), t \in D\}$ be centered and normed gaussian field defined on some domain $D \subset \mathbb{R}^{d}$. Suppose that $X$ is locally stationary of index $\alpha \in(0,2]$ with local structure $C_{t}(s)$. For $u>0$ set $q=q(u)=u^{-2 / \alpha}$. For $t=\left(t_{1}, \ldots, t_{d}\right) \in D$ set

$$
Q(t, u)=\left\{\left(s_{1}, \ldots, s_{d}\right) \mid s_{i} \in\left[t_{i}, t_{i}+q(u)\right], i=1, \ldots, d\right\} .
$$

Then, as $u \rightarrow \infty$,

$$
\mathbf{P}\left[\sup _{t \in Q(t, u)} X(t)>u\right] \sim H(t) \frac{1}{\sqrt{2 \pi}} \frac{1}{u} e^{-u^{2} / 2} .
$$

where $H(t) \in(0, \infty)$ is defined by

$$
H(t)=\lim _{T \rightarrow \infty} \frac{1}{T^{d}} \int_{0}^{\infty} \mathbf{P}\left[\sup _{s \in[0, T]^{d}} Y_{t}(s)>w\right] e^{w} d w .
$$

Here, $\left\{Y_{t}(s), s \in \mathbb{R}^{d}\right\}$ is the gaussian process defined by

$$
\mathbf{E}\left[Y_{t}(s)\right]=-C_{t}(s)
$$

and

$$
\operatorname{Cov}\left(Y_{t}\left(s_{1}\right), Y_{t}\left(s_{2}\right)\right)=C_{t}\left(s_{1}\right)+C_{t}\left(s_{2}\right)-C_{t}\left(s_{1}-s_{2}\right)
$$


The process $Y_{t}(\cdot)$ is called the tangent process of $X$ at point $t$ conditioned on $X(t)=\infty$. Note that the existence of the process $Y_{t}$ follows from Proposition 2.2.3.

The function $H(t)$ is called the high excursion intensity of the field $X$. Alternatively, $H(t)$ can be defined by

$$
H(t)=\lim _{T \rightarrow \infty} \frac{1}{T^{d}} \mathbf{E}\left[\exp \left(\sup _{s \in[0, T]^{d}} Y_{t}(s)\right)\right] .
$$

The next theorem describes the asymptotic behavior of the probability of high excursion of a locally stationary gaussian field. It corresponds to the second stage of Pickands' method.

Theorem 2.2.11 (see [42, 16]). Let $\{X(t), t \in D\}$ be a centered normed gaussian field defined on some domain $D \subset \mathbb{R}^{d}$. Suppose that its correlation function $r(\cdot, \cdot)$ satisfies $r\left(t_{1}, t_{2}\right)=1 \Leftrightarrow t_{1}=t_{2}$. Suppose that $X$ is locally stationary of order $\alpha$ with local structure $C_{t}(s)$. Let $K \subset D$ be a compact set with positive Jordan measure. Then, as $u \rightarrow \infty$,

$$
\mathbf{P}\left[\sup _{t \in K} X(t)>u\right] \sim \frac{1}{\sqrt{2 \pi}}\left(\int_{K} H(t) d t\right) u^{\frac{2 d}{\alpha}-1} e^{-u^{2} / 2},
$$

where the function $H(t): D \rightarrow(0, \infty)$ is the high excursion intensity of $X$ defined in Proposition 2.2.10.

We are interested in the following special case of the above theorem.

Corollary 2.2.12 (see [6, 16]). Let $\{X(t), t \in \mathbb{H}\}$ be the field of selfnormalized Brownian increments defined in Section 2.1. Let $K \subset \mathbb{H}$ be a compact set with positive Jordan measure. Then, as $u \rightarrow \infty$,

$$
\mathbf{P}\left[\sup _{t \in K} X(t)>u\right] \sim \frac{1}{4 \sqrt{2 \pi}} \int_{K} \frac{d x d y}{y^{2}} u^{3} e^{-u^{2} / 2} .
$$

\subsection{Proof of the Main Theorem}

In this section we prove Theorem 2.1.2. First, we introduce some notation. Let $\tau \in \mathbb{R}$ be fixed. Let $u=u(n)=a_{n}+b_{n} \tau$ with $a_{n}, b_{n}$ defined by (3.1), 3.2). Note that $u \rightarrow \infty$ as $n \rightarrow \infty$. 
Remark 2.3.1. We have, as $u, n \rightarrow \infty$,

$$
\frac{1}{4 \sqrt{2 \pi}} u^{3} e^{-u^{2} / 2} \sim e^{-\tau} / n
$$

Divide $\mathbb{H}$ into rectangles

$$
R_{k, l}=\left[2^{l+1} k, 2^{l+1}(k+1)\right] \times\left[2^{l}, 2^{l+1}\right], \quad k, l \in \mathbb{Z} .
$$

\section{Remark 2.3.2.}

$$
\int_{R_{k, l}} \frac{d x d y}{y^{2}}=1
$$

Remark 2.3.3. Let $k, l \in \mathbb{Z}$. Then, as $u, n \rightarrow \infty$,

$$
\mathbf{P}\left[\sup _{t \in R_{k, l}} X(t)>u\right] \sim \frac{e^{-\tau}}{n} .
$$

Proof. Follows directly from Corollary 2.2.12 and the previous remarks.

Let $H(n)$ be the triangle $\{(x, y) \in \mathbb{H} \mid x \in[0, n], y \in[1, n-x]\}$.

Let $H_{*}(n)$ be the union of all rectangles of the form $R_{k, l}$ which are inside $H(n)$ and $H^{*}(n)$ be the union of all rectangles which intersect the interior of $H(n)$. It is clear that $H_{*}(n) \subset H(n) \subset H^{*}(n)$. Note also that all three sets $H(n), H_{*}(n), H^{*}(n)$ consist of $n+o(n)$ rectangles.

Lemma 2.3.4. We have, as $u, n \rightarrow \infty$,

$$
\mathbf{P}\left[\sup _{t \in H_{*}(n)} X(t) \leq u\right]-\mathbf{P}\left[\sup _{t \in H(n)} X(t) \leq u\right] \rightarrow 0 .
$$

Proof. It follows from $H_{*}(n) \subset H(n)$ that

$$
\mathbf{P}\left[\sup _{t \in H_{*}(n)} X(t) \leq u\right]-\mathbf{P}\left[\sup _{t \in H(n)} X(t) \leq u\right] \geq 0
$$

The next inequality follows from $H_{*}(n) \subset H(n) \subset H^{*}(n)$

$$
\mathbf{P}\left[\sup _{t \in H_{*}(n)} X(t) \leq u\right]-\mathbf{P}\left[\sup _{t \in H(n)} X(t) \leq u\right] \leq \mathbf{P}\left[\sup _{t \in H^{*}(n) \backslash H_{*}(n)} X(t)>u\right]
$$


The last probability is smaller than

$$
\sum_{R_{k, l} \subset H^{*}(n) \backslash H_{*}(n)} \mathbf{P}\left[\sup _{t \in R_{k, l}} X(t)>u\right] .
$$

Now the sum has at most $o(n)$ (equal) terms and each term is $O(1 / n)$ by Remark 2.3.3. This finishes the proof.

From now on we write $H(n)$ instead of $H_{*}(n)$. Let $\varepsilon>0$. For each rectangle $R_{k, l}$ consider a smaller rectangle $R_{k, l}(\varepsilon)$ centered at the same point as $R_{k, l}$ and satisfying

$$
\int_{R_{k, l}(\varepsilon)} \frac{d x d y}{y^{2}}=1-\varepsilon
$$

Set

$$
H(n, \varepsilon)=\bigcup_{R_{k, l} \subset H(n)} R_{k, l}(\varepsilon)
$$

Lemma 2.3.5. We have, for some constant $c_{1}>0$ and every $\varepsilon \in(0,1)$

$$
0 \leq \limsup _{u \rightarrow \infty}\left(\mathbf{P}\left[\sup _{t \in H(n, \varepsilon)} X(t) \leq u\right]-\mathbf{P}\left[\sup _{t \in H(n)} X(t) \leq u\right]\right) \leq c_{1} \varepsilon .
$$

Proof. It follows from $H(n, \varepsilon) \subset H(n)$ that

$$
\mathbf{P}\left[\sup _{t \in H(n, \varepsilon)} X(t) \leq u\right]-\mathbf{P}\left[\sup _{t \in H(n)} X(t) \leq u\right] \geq 0,
$$

which proves the first part of the inequality. In order to prove the other part note that

$$
\mathbf{P}\left[\sup _{t \in H(n, \varepsilon)} X(t) \leq u\right]-\mathbf{P}\left[\sup _{t \in H(n)} X(t) \leq u\right] \leq \mathbf{P}\left[\sup _{t \in H(n) \backslash H(n, \varepsilon)} X(t)>u\right] .
$$

The last probability is smaller than

$$
\sum_{R_{k, l} \subset H(n)} \mathbf{P}\left[\sup _{t \in R_{k, l} \backslash R_{k, l}(\varepsilon)} X(t)>u\right] .
$$

Now the number of terms in the sum is $\sim n$. Actually, all terms are equal by the affine invariance. Each term is $\sim \varepsilon e^{-\tau} / n$ by Corollary 2.2.12. This proves the lemma. 
Let $a>0$. Define the following sets

$$
R_{k, l}(\varepsilon, a)=R_{k, l}(\varepsilon) \cap 2^{l} \frac{a}{u^{2}} \mathbb{Z}^{2} .
$$

Note that all these sets are finite and can be obtained from $R_{0,1}(\varepsilon, a)$ by dilation and translation. The next lemma is implicit in [16], the corresponding one-dimensional statement is proved in [36, Chapter 12].

Lemma 2.3.6. Let $X(t)$ be centered and normed gaussian field defined on some domain $D \subset \mathbb{R}^{d}$. Suppose $X$ is locally stationary of index $\alpha$. Let $K \subset D$ be a compact set with positive Jordan measure. Then

$0 \leq \limsup _{u \rightarrow \infty} \frac{1}{u^{\frac{2 d}{\alpha}-1} e^{-u^{2} / 2}}\left(\mathbf{P}\left[\sup _{t \in K \cap a u^{-2 / \alpha} \mathbb{Z}^{d}} X(t)>u\right]-\mathbf{P}\left[\sup _{t \in K} X(t)>u\right]\right) \leq \rho_{a}$.

for some sequence of constants $\left\{\rho_{a}, a>0\right\}$ with the property $\lim _{a \downarrow 0} \rho_{a}=0$.

Lemma 2.3.7. There are constants $\left\{\rho_{a}, a>0\right\}$ such that $\rho_{a} \rightarrow 0$ as $a \downarrow 0$ and

$$
\limsup _{u \rightarrow \infty} n\left(\mathbf{P}\left[\sup _{t \in R_{k, l}(\varepsilon, a)} X(t) \leq u\right]-\mathbf{P}\left[\sup _{t \in R_{k, l}(\varepsilon)} X(t) \leq u\right]\right) \leq \rho_{a} .
$$

Proof. This is a consequence of Lemma 2.3.6 and Remark 2.3.1.

Define

$$
H(n, \varepsilon, a)=\cup_{R_{k, l} \subset H(n)} R_{k, l}(\varepsilon, a) .
$$

\section{Lemma 2.3.8.}

$$
0 \leq \limsup _{u \rightarrow \infty}\left(\mathbf{P}\left[\sup _{t \in H(n, \varepsilon, a)} X(t) \leq u\right]-\mathbf{P}\left[\sup _{t \in H(n, \varepsilon)} X(t) \leq u\right]\right) \leq \rho_{a} .
$$

Proof. The lower estimate is evident, the upper estimate follows from the preceeding lemma.

Lemma 2.3.9.

$$
0 \leq \limsup _{u \rightarrow \infty}\left(\mathbf{P}\left[\sup _{t \in H(n, \varepsilon, a)} X(t) \leq u\right]-\mathbf{P}\left[\sup _{t \in H(n)} X(t) \leq u\right]\right) \leq \rho_{a}+c_{1} \varepsilon .
$$


Proof. Follows from Lemma 2.3.8 and Lemma 2.3.5.

The next lemma is known as Berman Inequality, see e.g. [36, Theorem 4.2.1].

Lemma 2.3.10 (Berman Inequality). Suppose $\xi_{1}, \ldots, \xi_{N}$ are standard normal variables with covariance matrix $\Lambda^{1}=\left(\Lambda_{i j}^{1}\right)$, and $\eta_{1}, \ldots, \eta_{N}$ similarly with covariance matrix $\Lambda^{2}=\left(\Lambda_{i j}^{2}\right)$, and let $\rho_{i j}=\max \left(\left|\Lambda_{i j}^{1}\right|,\left|\Lambda_{i j}^{2}\right|\right)$, $\delta=\max _{i \neq j} \rho_{i j}<1$. Then, for some constant $K$ depending only on $\delta$,

$\mathbf{P}\left[\max _{1 \leq i \leq N} \xi_{i} \leq u\right]-\mathbf{P}\left[\max _{1 \leq i \leq N} \eta_{i} \leq u\right] \leq K \sum_{1 \leq i<j \leq N}\left|\Lambda_{i j}^{1}-\Lambda_{i j}^{2}\right| \exp \left(-\frac{u^{2}}{2(1+\delta)}\right)$.

Let $\{Y(t), t \in H(n, \varepsilon, a)\}$ be standard normal variables with the following covariance matrix:

$$
\begin{aligned}
& \mathbf{E}\left[Y\left(t_{1}\right) Y\left(t_{2}\right)\right]=\mathbf{E}\left[X\left(t_{1}\right) X\left(t_{2}\right)\right] \quad \text { if } \exists k, l: t_{1}, t_{2} \in R_{k, l}(\varepsilon, a) \text {, } \\
& \mathbf{E}\left[Y\left(t_{1}\right) Y\left(t_{2}\right)\right]=0 \quad \text { otherwise. }
\end{aligned}
$$

Thus, we remove the dependence between $X\left(t_{1}\right)$ and $X\left(t_{2}\right)$ if $t_{1}$ and $t_{2}$ are in different $R_{k, l}$ 's.

\section{Lemma 2.3.11.}

$$
\lim _{u \rightarrow \infty}\left(\mathbf{P}\left[\max _{t \in H(n, \varepsilon, a)} X(t) \leq u\right]-\mathbf{P}\left[\max _{t \in H(n, \varepsilon, a)} Y(t) \leq u\right]\right)=0 .
$$

Proof. We use Berman Inequality for the variables $\{X(t), t \in H(n, \varepsilon, a)\}$ and $\{Y(t), t \in H(n, \varepsilon, a)\}$. It is easy to see that the correlations $\Lambda_{t_{1}, t_{2}}^{X}=$ $\mathbf{E}\left[X\left(t_{1}\right) X\left(t_{2}\right)\right]$ and $\Lambda_{t_{1}, t_{2}}^{Y}=\mathbf{E}\left[Y\left(t_{1}\right) Y\left(t_{2}\right)\right]\left(t_{1} \neq t_{2}\right)$ are bounded away from 1 by some constant $\delta$ depending on $\varepsilon$ and $a$, but not on $n$ (this is due to the affine invariance). We write $t_{1} \nsim t_{2}$ if $t_{1}$ and $t_{2}$ are contained in different sets $R_{k, l}(\varepsilon, a)$.

$$
\sum_{\substack{t_{1}, t_{2} \in H(n, \varepsilon, a) \\ t_{1} \neq t_{2}}}\left|\Lambda_{t_{1}, t_{2}}^{X}-\Lambda_{t_{1}, t_{2}}^{Y}\right|=\sum_{\substack{t_{1}, t_{2} \in H(n, \varepsilon, a) \\ t_{1} \nsim t_{2}}} \Lambda_{t_{1}, t_{2}}^{X} \leq \delta \sum_{\substack{t_{1}, t_{2} \in H(n, \varepsilon, a) \\ t_{1} \nsim t_{2}}} 1 .
$$

The number of elements of $R_{k, l}(\varepsilon, a)$ is less than $c_{2} u^{4}$, where the constant $c_{2}$ depends only on $a$. If $t_{1} \in R_{k, l}(\varepsilon, a)$ then an easy calculation shows that the number of $t_{2}$ with the property $t_{1} \nsim t_{2}$ is less than $c_{3} u^{4}\left(2^{l}+\log n\right)$. It 
follows that the number of pairs $\left(t_{1}, t_{2}\right)$ with the property $t_{1} \nsim t_{2}$ is less than $c_{4} n \log ^{3} n$. It follows from the Berman Inequality that

$$
\mathbf{P}\left[\max _{t \in H(n, \varepsilon, a)} X(t) \leq u\right]-\mathbf{P}\left[\max _{t \in H(n, \varepsilon, a)} Y(t) \leq u\right] \leq c_{5} n\left(\log ^{4} n\right) e^{-u^{2} /(1+\delta)} .
$$

where $c_{5}$ depends on $\varepsilon$ and $a$, but not on $n$. Recall that $u \sim \sqrt{2 \log n}$. The statement of the lemma follows.

\section{Lemma 2.3.12.}

$$
\limsup _{a \rightarrow 0} \limsup _{\varepsilon \rightarrow 0} \limsup _{u \rightarrow \infty}\left|\mathbf{P}\left[\max _{t \in H(n, \varepsilon, a)} Y(t) \leq u\right]-e^{-e^{-\tau}}\right|=0 .
$$

Proof. First let $\varepsilon, a$ be fixed and let $u, n \rightarrow \infty$. By Lemma 2.3.7

$$
\left|\mathbf{P}\left[\max _{t \in R_{0,1}(\varepsilon, a)} X(t)>u\right]-\mathbf{P}\left[\max _{t \in R_{0,1}(\varepsilon)} X(t)>u\right]\right| \leq \rho_{a} \frac{1}{n}(1+o(1)) .
$$

and by Corollary 2.2 .12

$$
\mathbf{P}\left[\max _{t \in R_{0,1}(\varepsilon)} X(t)>u\right]=(1-\varepsilon) e^{-\tau} \frac{1}{n}(1+o(1)) .
$$

It follows that

$$
\liminf _{u \rightarrow \infty} n \mathbf{P}\left[\max _{t \in R_{0,1}(\varepsilon, a)} X(t)>u\right] \geq e^{-\tau}-c_{1}(\varepsilon, a)
$$

and

$$
\limsup _{u \rightarrow \infty} n \mathbf{P}\left[\max _{t \in R_{0,1}(\varepsilon, a)} X(t)>u\right] \leq e^{-\tau}+c_{2}(\varepsilon, a),
$$

where $c_{1}(\varepsilon, a) \rightarrow 0$ and $c_{2}(\varepsilon, a) \rightarrow 0$ as $\varepsilon, a \rightarrow 0$. Since $Y\left(t_{1}\right)$ and $Y\left(t_{2}\right)$ are independent if $t_{1}$ and $t_{2}$ are in different $R_{k, l}$ 's, we have

$$
\begin{aligned}
\mathbf{P}\left[\max _{t \in H(n, \varepsilon, a)} Y(t) \leq u\right]= & \left(1-\mathbf{P}\left[\max _{t \in R_{0,1}(\varepsilon, a)} Y(t)>u\right]\right)^{n+o(n)}= \\
& \left(1-\mathbf{P}\left[\max _{t \in R_{0,1}(\varepsilon, a)} X(t)>u\right]\right)^{n+o(n)} .
\end{aligned}
$$


By letting $u, n \rightarrow \infty$ we see that

$$
\limsup _{u \rightarrow \infty} \mathbf{P}\left[\max _{t \in H(n, \varepsilon, a)} Y(t) \leq u\right] \leq \exp \left(-e^{-\tau}+c_{1}(\varepsilon, a)\right)
$$

and

$$
\liminf _{u \rightarrow \infty} \mathbf{P}\left[\max _{t \in H(n, \varepsilon, a)} Y(t) \leq u\right] \geq \exp \left(-e^{-\tau}-c_{2}(\varepsilon, a)\right) .
$$

Now let $a, \varepsilon \rightarrow 0$. The lemma follows.

Proof of Theorem 2.1.2. Follows from Lemmas 2.3.9, 2.3.11, 2.3.12. 


\section{Chapter 3}

\section{Extreme-Value Analysis of Self-Normalized Totally Skewed $\alpha$-stable Increments}

\subsection{Introduction}

In this chapter we prove the following

Theorem 3.1.1. Let $\{\xi(x), x \geq 0\}$ be a stable Lévy process of index $\alpha \in(1,2]$ with skewness parameter $\beta=-1$. For $n>1$ set

$$
L_{n}=\sup _{\substack{x_{1}, x_{2} \in[0,1] \\ x_{2}-x_{1} \geq 1 / n}} \frac{\xi\left(x_{2}\right)-\xi\left(x_{1}\right)}{\left(x_{2}-x_{1}\right)^{1 / \alpha}} .
$$

Then, for every $\tau \in \mathbb{R}$,

$$
\lim _{n \rightarrow \infty} \mathbf{P}\left[L_{n} \leq a_{n}+b_{n} \tau\right]=\exp \left(-e^{-\tau}\right),
$$

where the constants $a_{n}$ and $b_{n}$ are given by

$$
\begin{aligned}
a_{n} & =\frac{1}{B_{\alpha}^{(\alpha-1) / \alpha}}\left((\log n)^{(\alpha-1) / \alpha}+\frac{\alpha-1}{\alpha} \frac{3 / 2 \log _{2} n-\log \sqrt{2 \alpha \pi}}{(\log n)^{1 / \alpha}}\right), \\
b_{n} & =\frac{1}{B_{\alpha}^{(\alpha-1) / \alpha}} \frac{\alpha-1}{\alpha} \frac{1}{(\log n)^{1 / \alpha}} .
\end{aligned}
$$

Here,

$$
B_{\alpha}=(\alpha-1) \alpha^{-\alpha /(\alpha-1)}|\cos (\pi \alpha / 2)|^{1 /(\alpha-1)} .
$$


We fix some notation which will be used throughout this chapter. Let

$$
\mathbb{H}=\left\{t=(x, y) \in \mathbb{R}^{2} \mid y>0\right\}
$$

denote the open upper half-plane.

Let $\{\xi(x), x \geq 0\}$ be a stable Lévy process of index $\alpha \in(1,2)$ with skewness parameter $\beta=-1$. Some neccesary facts about stable distributions and stable Lévy processes will be recalled in Section 3.2.

Define a random field $\{X(t), t=(x, y) \in \mathbb{H}\}$ by

$$
X(t)=\frac{\xi(x+y)-\xi(x)}{y^{1 / \alpha}} .
$$

Note that for each $t \in \mathbb{H}$ the distribution of $X(t)$ is standard $\alpha$-stable with skewness $\beta=-1$ (i.e. it coincides with the distribution of $\xi(1)$ ).

As in Section 2.1, one defines the natural action of the one-dimensional affine group on $\mathbb{H}$ and obtains, using the scaling property of stable Lévy processes (see Proposition 3.2 .12 below) the following invariance property of the field $X$, which will be often used in the sequel.

Proposition 3.1.2. Let $g$ be an affine transformation of $\mathbb{R}$. Then, for each $t_{1}, \ldots, t_{n} \in \mathbb{H}$, the joint distribution of $X\left(g\left(t_{1}\right)\right), \ldots, X\left(g\left(t_{n}\right)\right)$ coincides with the joint distribution of $X\left(t_{1}\right), \ldots, X\left(t_{n}\right)$.

Thus, we may state Theorem 3.1.1 in the following, equivalent form.

Theorem 3.1.3. For $n>1$ let $H(n)$ be the triangle $\{(x, y) \in \mathbb{H} \mid x \in$ $[0, n], y \in[1, n-x]\}$. Define $X$ by (3.3). Then, for every $\tau \in \mathbb{R}$,

$$
\lim _{n \rightarrow \infty} \mathbf{P}\left[\sup _{t \in G_{n}} X(t) \leq a_{n}+b_{n} \tau\right]=\exp \left(-e^{-\tau}\right),
$$

where $a_{n}, b_{n}$ are defined by 3.1) and (3.2).

The rest of the chapter is devoted to the proof of Theorem 3.1.3.

In Section 3.2, we recall some well-known facts about stable distributions and processes.

In Section 3.3 , we investigate the probability of the presence of a high excursion of $X$ over an infinitesimally small domain (the first stage of Pickands' method). 
In Section 3.4, we prove a result about the distribution of the maximum of an $\alpha$-stable totally skewed process with negative drift. The main result of this section, Theorem 3.4.3, will be needed in the next section.

In Section 3.5, we investigate the high excursion probability over a compact set of finite positive Jordan measure. The main result of this section corresponds to Corollary 2.2.12 of Chapter 2 (the second stage of Pickands' method).

In Section 3.6, we finish the proof (the third stage of Pickands' method).

\subsection{Stable Lévy Processes}

We recall here some facts about stable distributions and processes needed in the sequel. See [51], [32] or [55] for more information.

Definition 3.2.1. A random variable $\xi$ is said to have a stable distribution $S_{\alpha}(\sigma, \beta, \mu)$ with parameters $\alpha \in(0,2], \sigma \geq 0, \beta \in[-1,1]$ and $\mu \in \mathbb{R}$ if its characteristic function has the following form:

$$
\begin{array}{ll}
\mathbf{E} \exp (i q \xi)=\exp \left(-\sigma^{\alpha}|q|^{\alpha}(1-i \beta \operatorname{sign}(q) \tan (\pi \alpha / 2)+i \mu q)\right) & \text { if } \alpha \neq 1, \\
\mathbf{E} \exp (i q \xi)=\exp \left(-\sigma|q|\left(1+i \beta \frac{2}{\pi} \operatorname{sign}(q) \log |q|\right)+i \mu q\right) & \text { if } \alpha=1 .
\end{array}
$$

Definition 3.2.2. A variable $\xi \sim S_{\alpha}(\sigma, \beta, \mu)$ is said to be standard $\alpha$-stable with skewness $\beta$ if $\sigma=1$ and $\mu=0$.

Definition 3.2.3. A variable $\xi \sim S_{\alpha}(\sigma, \beta, \mu)$ is called totally skewed to the left if $\beta=-1$ and totally skewed to the right if $\beta=1$.

Proposition 3.2.4. The support of the standard $\alpha$-stable distribution with skewness $\beta$ is

$$
\begin{array}{ll}
{[0,+\infty]} & \text { if } \alpha \in(0,1) \text { and } \beta=+1, \\
{[-\infty, 0]} & \text { if } \alpha \in(0,1) \text { and } \beta=-1, \\
\mathbb{R} & \text { otherwise. }
\end{array}
$$

Although the support of the standard stable distribution with $\alpha \in(1,2)$, $\beta= \pm 1$ is the whole $\mathbb{R}$, one can still define its Laplace transform. 
Proposition 3.2.5. Let $\xi$ be a standard $\alpha$-stable random variable with skewness parameter $\beta=-1$. The Laplace transform $\mathbf{E}[\exp (q \xi)]$ exists for $q \geq 0$ and is given by

$$
\begin{array}{ll}
\mathbf{E}[\exp (q \xi)]=\exp \left(\frac{q^{\alpha}}{|\cos (\pi \alpha / 2)|}\right) & \text { if } \alpha \in(0,1) \cup(1,2), \\
\mathbf{E}[\exp (q \xi)]=\exp \left(\frac{2}{\pi} q \log q\right) & \text { if } \alpha=1 .
\end{array}
$$

Proposition 3.2.6. If $\xi_{1} \sim S_{\alpha}\left(\sigma_{1}, \beta, 0\right)$ and $\xi_{2} \sim S_{\alpha}\left(\sigma_{2}, \beta, 0\right)$ are independent then

$$
\xi_{1}+\xi_{2} \sim S_{\alpha}(\sigma, \beta, 0)
$$

where $\sigma=\left(\sigma_{1}^{\alpha}+\sigma_{2}^{\alpha}\right)^{1 / \alpha}$.

Proposition 3.2.7. Let $\xi \sim S_{\alpha}(\sigma, \beta, \mu)$ and $a>0$. Then

$$
\begin{array}{lll}
a \xi & \sim S_{\alpha}(a \sigma, \beta, a \mu) & \text { if } \alpha \neq 1, \\
a \xi \sim S_{1}\left(a \sigma, \beta, a \mu-\frac{2}{\pi} a \log (a) \sigma \beta\right) & \text { if } \alpha=1 .
\end{array}
$$

Now we recall the definition of stable Lévy processes.

Definition 3.2.8. Let $\alpha \in(0,2]$ and $\beta \in[-1,1]$. A stochastic process $\{\xi(t), t \geq 0\}$ is called (standard) $\alpha$-stable Lévy process with skewness parameter $\beta$ if

1) $\xi(0)=0$.

2) $\xi$ has independent increments.

3) $\xi(t)-\xi(s) \sim S_{\alpha}\left((t-s)^{1 / \alpha}, \beta, 0\right)$ for any $0 \leq s<t<\infty$.

If $\alpha=2$, we obtain $\sqrt{2} B(t)$ where $\{B(t), t \geq 0\}$ is the standard Brownian motion.

Proposition 3.2.9. Any stable Lévy process admits a càdlàg modification.

In the sequel, we always assume that the process under consideration has càdlàg sample paths.

If $\beta=+1$ (resp. $\beta=-1$ ) the process is called totally skewed to the right (resp. to the left). 
Proposition 3.2.10. Let $\{\xi(x), x \geq 0\}$ be an $\alpha$-stable Lévy process totally skewed to the right, where $\alpha \in(0,1)$. Then $\xi$ has increasing sample paths and is called the $\alpha$-stable subordinator.

In the sequel, we shall consider only stable Lévy processes with $\alpha \in(1,2)$ and $\beta=-1$. These processes have the following property.

Proposition 3.2.11. Let $\xi$ be an $\alpha$-stable process with $\alpha \in[1,2], \beta=-1$. Then the sample paths of $\xi$ have no positive jumps meaning that

$$
\mathbf{P}\left[\exists t>0 \mid \xi_{+}(t)-\xi_{-}(t)>0\right]=0,
$$

where

$$
\xi_{+}(t)=\lim _{s \rightarrow t+0} \xi(s), \quad \xi_{-}(t)=\lim _{s \rightarrow t-0} \xi(s) .
$$

Recall also the scaling property of stable Lévy processes.

Proposition 3.2.12. Let $\{\xi(t), t \geq 0\}$ be an $\alpha$-stable Lévy process with skewness parameter $\beta$ and let $a>0$. If $\alpha \neq 1$, the rescaled process $\xi($ at) has the same finite-dimensional distributions as $a^{1 / \alpha} \xi(t)$. If $\alpha=1$, the rescaled process $\xi(a t)$ has the same finite-dimensional distributions as $a \xi(t)+\frac{2}{\pi} \beta t \log a$.

\subsection{Proof of Theorem 3.1.1: Infinitesimal Max- ima}

In this section we prove an asymptotic relation for the probability of high excursion of the random field $X$ over an infinitesimally small set. More precisely, we study the asymptotic behavior as $u \rightarrow \infty$ of the probability

$$
\mathbf{P}\left[\sup _{Q} X(t)>u\right],
$$

where $Q=Q(u)$ is a small parallelogram whose area tends to 0 as $u \rightarrow \infty$. We start by recalling the following well-known fact about the tail behavior of totally-skewed stable distributions (see e.g. [32], [55] or [2]).

Lemma 3.3.1. Let $\xi$ be a standard stable variable of index $\alpha \in(1,2)$ with skewness parameter $\beta=-1$. Write $\Phi(u)$ and $f(u)$ for the distribution function and density of $\xi$ respectively. Then, as $u \rightarrow \infty$,

$$
\begin{aligned}
1-\Phi(u) & \sim(\alpha-1) A_{\alpha} u^{-\alpha /(2(\alpha-1))} \exp \left(-B_{\alpha} u^{\alpha /(\alpha-1)}\right), \\
f(u) & \sim \alpha A_{\alpha} B_{\alpha} u^{(2-\alpha) /(2(\alpha-1))} \exp \left(-B_{\alpha} u^{\alpha /(\alpha-1)}\right)
\end{aligned}
$$


where

$$
A_{\alpha}=\left(2 \pi(\alpha-1)^{3}\right)^{-1 / 2} \alpha^{1 /(2 \alpha-2)}|\cos (\pi \alpha / 2)|^{-1 /(2 \alpha-2)}
$$

and

$$
B_{\alpha}=(\alpha-1) \alpha^{-\alpha /(\alpha-1)}|\cos (\pi \alpha / 2)|^{1 /(\alpha-1)} .
$$

Corollary 3.3.2. For some constants $C_{1}, C_{2}>0$

$$
1-\Phi(u)<C_{1} \exp \left(-C_{2} u^{\alpha /(\alpha-1)}\right), \quad u>0 .
$$

It is known (see [15]) that the supremum of a totally skewed $\alpha$-stable Lévy process over the interval $[0,1]$ has Mittag-Leffler distribution with parameter $1 / \alpha$. We shall need only the following consequence of this fact.

Proposition 3.3.3. Let $\{\xi(x), x \geq 0\}$ be a stable Lévy process of index $\alpha \in(1,2)$ with skewness parameter $\beta=-1$. Then, for some constant $C_{\alpha}$,

$$
\mathbf{P}\left[\sup _{x \in[0,1]} \xi(x)>u\right] \sim C_{\alpha} \mathbf{P}[\xi(1)>u] .
$$

Note that Proposition 3.3.3 was also proved in [2] using the method of double sums.

Define $w=w(u)$ and $q=q(u)$ by

$$
w=\frac{\alpha-1}{\alpha B_{\alpha}} u^{-1 /(\alpha-1)}
$$

and

$$
q=w^{\alpha}
$$

Note that $q(u), w(u) \rightarrow 0$ as $u \rightarrow \infty$.

The next two lemmas are standard in the extreme-value theory and can be proved by an elementary calculation.

Lemma 3.3.4. Let $z \in \mathbb{R}$ be fixed. Then

$$
\lim _{u \rightarrow \infty} \frac{1-\Phi(u-w z)}{1-\Phi(u)}=e^{z} .
$$

Lemma 3.3.5. Let $z \in \mathbb{R}$ be fixed. Then

$$
\lim _{u \rightarrow \infty} \frac{w f(u-w z)}{1-\Phi(u)}=e^{z} .
$$


The next lemma will be needed later in order to justify the use of the dominated convergence theorem.

Lemma 3.3.6. For every $\varepsilon>0$ there is a constant $C_{1}$, such that for every $u>1$ and every $z>0$

$$
\frac{w f(u-w z)}{1-\Phi(u)}<C_{1} \exp \left(z^{1+\varepsilon}\right)
$$

Proof. Let $\delta>0$. Suppose first that

$$
z>u^{(1-\delta) \frac{\alpha}{\alpha-1}} .
$$

It follows from Corollary 3.3.2 that

$$
1-\Phi(u)>C_{2} \exp \left(-C_{3} u^{\alpha /(\alpha-1)}\right) .
$$

Since the density $f$ is bounded, we have

$$
\frac{w f(u-w z)}{1-\Phi(u)}<C_{4} \exp \left(C_{3} u^{\alpha /(\alpha-1)}\right)<C_{4} \exp \left(C_{3} z^{1 /(1-\delta)}\right) .
$$

Now let $\delta$ be so small that $1 /(1-\delta)<1+\varepsilon$. It follows that

$$
\frac{w f(u-w z)}{1-\Phi(u)}<C_{5} \exp \left(z^{1+\varepsilon}\right)
$$

Suppose now that

$$
z \leq u^{(1-\delta) \frac{\alpha}{\alpha-1}}
$$

Recall that $w=$ const $\cdot u^{-1 /(\alpha-1)}$. Note that

$$
\frac{w z}{u}<\mathrm{const} \cdot u^{-1 /(\alpha-1)} u^{(1-\delta) \frac{\alpha}{\alpha-1}} u^{-1}<C_{6} u^{-\varepsilon} .
$$

if $\delta$ is sufficiently small. It follows from Lemma 3.3.1 that

$$
\begin{aligned}
w f(u-w z)< & C_{7} u^{-\alpha /(2(\alpha-1))} \exp \left(-B_{\alpha}(u-w z)^{\alpha /(\alpha-1)}\right)= \\
& C_{7} u^{-\alpha /(2(\alpha-1))} \exp \left(-B_{\alpha} u^{\alpha /(\alpha-1)}(1-w z / u)^{\alpha /(\alpha-1)}\right) .
\end{aligned}
$$

By the Bernoulli inequality this is smaller than

$$
\begin{aligned}
& C_{7} u^{-\alpha /(2(\alpha-1))} \exp \left(-B_{\alpha} u^{\alpha /(\alpha-1)}\left(1-\frac{\alpha}{\alpha-1} w z / u\right)\right)= \\
& C_{7} u^{-\alpha /(2(\alpha-1))} \exp \left(-B_{\alpha} u^{\alpha /(\alpha-1)}\right) \exp \left(C_{8} u^{\alpha /(\alpha-1)} u^{-1 /(\alpha-1)} z u^{-1}\right)= \\
& C_{7} u^{-\alpha /(2(\alpha-1))} \exp \left(-B_{\alpha} u^{\alpha /(\alpha-1)}\right) \exp \left(C_{8} z\right) .
\end{aligned}
$$


Using this and Lemma 3.3.1 we obtain

$$
\frac{w f(u-w z)}{1-\Phi(u)}<\frac{C_{7} u^{-\alpha /(2(\alpha-1))} \exp \left(-B_{\alpha} u^{\alpha /(\alpha-1)}\right) \exp \left(C_{8} z\right)}{C_{8} u^{-\alpha /(2(\alpha-1))} \exp \left(-B_{\alpha} u^{\alpha /(\alpha-1)}\right)}=C_{9} e^{C_{8} z},
$$

which is smaller than $C \exp \left(z^{1+\varepsilon}\right)$.

This finishes the proof of the lemma.

Take $n \in \mathbb{N}$. Let $t_{0}=\left(x_{0}, y_{0}\right) \in \mathbb{H}$ be fixed. Define

$Q\left(t_{0}, u, n\right)=\left\{\left(x_{0}-s_{x}, y_{0}+s_{x}+s_{y}\right) \in \mathbb{H} \mid 0 \leq s_{x} \leq n q(u), 0 \leq s_{y} \leq n q(u)\right\}$.

Note that $Q\left(t_{0}, u, n\right)$ is a paralellogram of area $n^{2} q^{2}$.

Let $\left\{\xi_{1}(a), a \geq 0\right\}$ and $\left\{\xi_{2}(b), b \geq 0\right\}$ be two independent stable Lévy processes of index $\alpha$ with skewness parameter $\beta=-1$. Define a process $\left\{Y\left(s_{x}, s_{y}\right), s_{x} \geq 0, s_{y} \geq 0\right\}$ by

$$
Y\left(s_{x}, s_{y}\right)=\xi_{1}\left(s_{x}\right)+\xi_{2}\left(s_{y}\right)-|\cos (\pi \alpha / 2)|^{-1}\left(s_{x}+s_{y}\right) .
$$

Lemma 3.3.7. Let $z>0$. Then

$\lim _{u \rightarrow \infty} \mathbf{P}\left[\sup _{t \in Q\left(t_{0}, u, n\right)} X(t)>u \mid X\left(t_{0}\right)=u-w z\right]=\mathbf{P}\left[\max _{s_{x}, s_{y} \in\left[0, n / y_{0}\right]} Y\left(s_{x}, s_{y}\right)>z\right]$.

Proof. Define

$$
P(u)=\mathbf{P}\left[\sup _{t \in Q\left(t_{0}, u, n\right)} X(t)>u \mid X\left(t_{0}\right)=u-w z\right] .
$$

Under the condition $X\left(t_{0}\right)=u-w z$ we have for $t=\left(x_{0}-s_{x}, y_{0}+s_{x}+s_{y}\right) \in$ $Q\left(t_{0}, u, n\right)$

$$
X(t)=\frac{(u-w z) y_{0}^{1 / \alpha}+\left(\xi\left(x_{0}\right)-\xi\left(x_{0}-s_{x}\right)\right)+\left(\xi\left(x_{0}+y_{0}+s_{y}\right)-\xi\left(x_{0}+y_{0}\right)\right)}{\left(y_{0}+s_{x}+s_{y}\right)^{1 / \alpha}}
$$

Note that the joint distribution of $\xi\left(x_{0}\right)-\xi\left(x_{0}-s_{x}\right)$ and $\xi\left(x_{0}+y_{0}+s_{y}\right)-$ $\xi\left(x_{0}+y_{0}\right)$ coincides with the joint distribution of $\xi_{1}\left(s_{x}\right)$ and $\xi_{2}\left(s_{y}\right)$. Using this fact we obtain

$$
P(u)=\mathbf{P}\left[\sup _{0 \leq s_{x}, s_{y} \leq n q} \frac{(u-w z) y_{0}^{1 / \alpha}+\xi_{1}\left(s_{x}\right)+\xi_{2}\left(s_{y}\right)}{\left(y_{0}+s_{x}+s_{y}\right)^{1 / \alpha}}>u\right]=
$$


or

$$
P(u)=\mathbf{P}\left[\sup _{0 \leq s_{x}, s_{y} \leq n q} \frac{(u-w z)+y_{0}^{-1 / \alpha} \xi_{1}\left(s_{x}\right)+y_{0}^{-1 / \alpha} \xi_{2}\left(s_{y}\right)}{\left(1+\frac{s_{x}+s_{y}}{y_{0}}\right)^{1 / \alpha}}>u\right] .
$$

Or, by introducing the new variables $p_{x}=s_{x} /\left(q y_{0}\right), p_{y}=s_{y} /\left(q y_{0}\right)$,

$$
P(u)=\mathbf{P}\left[\sup _{0 \leq p_{x}, p_{y} \leq n / y_{0}} \frac{(u-w z)+y_{0}^{-1 / \alpha} \xi_{1}\left(p_{x} q y_{0}\right)+y_{0}^{-1 / \alpha} \xi_{2}\left(p_{y} q y_{0}\right)}{\left(1+p_{x} q+p_{y} q\right)^{1 / \alpha}}>u\right]
$$

Using the scaling property of stable processes (see Proposition 3.2.12 and elementary transformations, we obtain

$P(u)=\mathbf{P}\left[\sup _{0 \leq p_{x}, p_{y} \leq n / y_{0}} \xi_{1}\left(p_{x}\right)+\xi_{2}\left(p_{y}\right)>q^{-1 / \alpha}\left(u\left(1+q\left(p_{x}+p_{y}\right)\right)^{1 / \alpha}-(u-w z)\right)\right]$.

But a simple calculation shows that

$$
\lim _{u \rightarrow \infty} q^{-1 / \alpha}\left(u\left(1+q\left(p_{x}+p_{y}\right)\right)^{1 / \alpha}-(u-w z)\right)=|\cos (\pi \alpha / 2)|^{-1}\left(p_{x}+p_{y}\right)+z .
$$

Thus

$$
\lim _{u \rightarrow \infty} P(u)=\mathbf{P}\left[\sup _{0 \leq p_{x}, p_{y} \leq n / y_{0}} \xi_{1}\left(p_{x}\right)+\xi_{2}\left(p_{y}\right)-|\cos (\pi \alpha / 2)|^{-1}\left(p_{x}+p_{y}\right)>z\right] .
$$

Recalling the definition of $Y\left(s_{x}, s_{y}\right)$ we obtain

$$
\lim _{u \rightarrow \infty} P(u)=\mathbf{P}\left[\sup _{0 \leq s_{x}, s_{y} \leq n / y_{0}} Y\left(s_{x}, s_{y}\right)>z\right]
$$

This finishes the proof.

The next lemma will be needed for the justification of the use of the dominated convergence theorem.

Lemma 3.3.8. There are constants $C_{1}$ and $C_{2}$ such that the following inequality holds for all $z>0, u>1$

$$
\mathbf{P}\left[\sup _{t \in Q\left(t_{0}, u, n\right)} X(t)>u \mid X\left(t_{0}\right)=u-w z\right]<C_{1} \exp \left(-C_{2} z^{\alpha /(\alpha-1)}\right) .
$$


Proof. Define

$$
P(u)=\mathbf{P}\left[\sup _{t \in Q\left(t_{0}, u, n\right)} X(t)>u \mid X\left(t_{0}\right)=u-w z\right]
$$

Then, as in the previous lemma,

$P(u)=\mathbf{P}\left[\sup _{0 \leq s_{x}, s_{y} \leq n / y_{0}} \xi_{1}\left(s_{x}\right)+\xi_{2}\left(s_{y}\right)>q^{-1 / \alpha}\left(u\left(1+q\left(s_{x}+s_{y}\right)\right)^{1 / \alpha}-(u-w z)\right)\right]$.

Note that

$$
q^{-1 / \alpha}\left(u\left(1+q\left(s_{x}+s_{y}\right)\right)^{1 / \alpha}-(u-w z)\right) \geq q^{-1 / \alpha} w z=z .
$$

It follows that

$$
P(u) \leq \mathbf{P}\left[\sup _{0 \leq s_{x}, s_{y} \leq n / y_{0}} \xi_{1}\left(s_{x}\right)+\xi_{2}\left(s_{y}\right)>z\right] \leq 2 \mathbf{P}\left[\sup _{0 \leq s_{x} \leq n / y_{0}} \xi_{1}\left(s_{x}\right)>z / 2\right] .
$$

By the scaling property (see Proposition 3.2.12) this is equal to

$$
2 \mathbf{P}\left[\sup _{0 \leq s_{x} \leq 1} \xi_{1}\left(s_{x}\right)>\left(n / y_{0}\right)^{-1 / \alpha} z / 2\right]
$$

It follows from Proposition 3.3.3 that this is smaller than

$$
C_{1} \exp \left(-C_{2} z^{\alpha /(\alpha-1)}\right) .
$$

This finishes the proof.

The following proposition is the main result of this section.

Proposition 3.3.9. The following relation holds for the probability of high excursion over the square $Q\left(t_{0}, u, n\right)$

$$
\lim _{u \rightarrow \infty} \frac{1}{1-\Phi(u)} \mathbf{P}\left[\max _{t \in Q\left(t_{0}, u, n\right)} X(t)>u\right]=G\left(n / y_{0}\right),
$$

where

$$
G(n)=\mathbf{E}\left[\exp \left(\sup _{0 \leq s_{x}, s_{y} \leq n} Y\left(s_{x}, s_{y}\right)\right)\right] .
$$


Proof. It is easy to see that

$$
\begin{aligned}
& \mathbf{P}\left[\max _{t \in Q\left(t_{0}, u, n\right)} X(t)>u\right]=\mathbf{P}\left[X\left(t_{0}\right)>u\right]+ \\
& \quad \int_{0}^{\infty} \mathbf{P}\left[\sup _{t \in Q\left(t_{0}, u, n\right)} X(t)>u \mid X\left(t_{0}\right)=u-w z\right] f(u-w z) w d z .
\end{aligned}
$$

Recall that $\mathbf{P}\left[X\left(t_{0}\right)>u\right]=1-\Phi(u)$. It follows that

$$
\begin{aligned}
& \frac{1}{1-\Phi(u)} \mathbf{P}\left[\max _{t \in Q\left(t_{0}, u, n\right)} X(t)>u\right]= \\
& 1+\int_{0}^{\infty} \mathbf{P}\left[\max _{t \in Q\left(t_{0}, u, n\right)} X(t)>u \mid X\left(t_{0}\right)=u-w z\right] \frac{f(u-w z) w}{1-\Phi(u)} d z .
\end{aligned}
$$

Since we have by Lemmas 3.3.7 and 3.3.5

$$
\lim _{u \rightarrow \infty} \mathbf{P}\left[\max _{t \in Q\left(t_{0}, u, n\right)} X(t)>u \mid X\left(t_{0}\right)=u-w z\right]=\mathbf{P}\left[\sup _{0 \leq s_{x}, s_{y} \leq n} Y\left(s_{x}, s_{y}\right)>z\right]
$$

and

$$
\lim _{u \rightarrow \infty} \frac{f(u-w z) w}{1-\Phi(u)}=e^{z},
$$

we obtain, at least formally,

$\lim _{u \rightarrow \infty} \frac{1}{1-\Phi(u)} \mathbf{P}\left[\max _{t \in Q\left(t_{0}, u, n\right)} X(t)>u\right]=1+\int_{0}^{\infty} \mathbf{P}\left[\sup _{0 \leq s_{x}, s_{y} \leq n} Y\left(s_{x}, s_{y}\right)>z\right] e^{z} d z$

In order to justify the use of the dominated convergence theorem note that by Lemmas 3.3.6 and 3.3.8

$$
\frac{w f(u-w z)}{1-\Phi(u)}<C(\varepsilon) \exp \left(z^{1+\varepsilon}\right), \quad \forall \varepsilon>0
$$

and

$$
\mathbf{P}\left[\sup _{t \in R\left(t_{0}, u, n\right)} X(t)>u \mid X\left(t_{0}\right)=u-w z\right]<C_{1} \exp \left(-C_{2} z^{\alpha /(\alpha-1)}\right) .
$$

It remains to note that $\alpha /(\alpha-1)>1$ (recall that $1<\alpha<2$ ). 


\subsection{The Maximum of a Drifted Totally Skewed Stable Process}

We recall some facts from the theory of spectrally negative Lévy processes (see [13, Chapter VII ] for more information).

A Lévy process is called spectrally negative if its Lévy measure is concentrated on $[-\infty, 0]$. For our purposes, the following example suffices: if $\{\xi(x), x \geq 0\}$ is a stable Lévy process with $\alpha \in(1,2)$ and $\beta=-1$, then the drifted process $\eta(x)=\xi(x)-\mu x$ is spectrally negative for $\mu \geq 0$. It is known that for a spectrally negative Lévy process $\{\eta(x), x \geq 0\}$ the Laplace transform $\mathbf{E}[\exp (\lambda \eta(x))]$ exists for $\lambda \geq 0$ and can be represented in the form $\exp (x \psi(\lambda))$ for some function $\psi:[0, \infty) \rightarrow \mathbb{R}$. In the case $\eta(x)=\xi(x)-\mu x$ we have

$$
\psi(\lambda)=|\cos (\pi \alpha / 2)|^{-1} \lambda^{\alpha}-\mu \lambda .
$$

Let $\psi^{-1}(0)$ be the largest solution of the equation $\psi(\lambda)=0$. Note that $\psi^{-1}(0) \geq 0$. Let $\psi^{-1}:[0, \infty) \rightarrow\left[\psi^{-1}(0), \infty\right)$ denote the inverse function of $\psi$. We need the following proposition from [13]

Theorem 3.4.1. Let $\{\eta(x), x>0\}$ be a spectrally negative Lévy process. Define $\psi, \psi^{-1}$ as above. Let $\tau(q)$ be an exponential random variable with parameter $q>0$ independent of $\eta$. Then $\sup _{x \in[0, \tau(q)]} \eta(x)$ is exponentially distributed with parameter $\psi^{-1}(q)$. Furthermore, if the derivative $\psi^{\prime}(0+)$ is negative then the process drifts to $-\infty$ and $\sup _{x>0} \eta(x)$ is exponentially distributed with parameter $\psi^{-1}(0)$. Otherwise, $\sup _{x \geq 0} \eta(x)$ is infinite a.s.

We obtain the following corollary, proved also in [47].

Corollary 3.4.2. Let $\{\xi(x), x \geq 0\}$ be a stable Lévy process of index $\alpha \in$ $(1,2]$ with skewness parameter $\beta=-1$. Let $\mu>0$. Then

$$
\sup _{x \geq 0}(\xi(x)-\mu x)
$$

is exponentially distributed with parameter $(\mu|\cos (\pi \alpha / 2)|)^{1 /(\alpha-1)}$.

The above corollary shows that for $\mu=|\cos (\pi \alpha / 2)|^{-1}$

$$
\mathbf{E} \exp \left(\sup _{x \geq 0}(\xi(x)-\mu x)\right)=+\infty .
$$

This motivates partially the following theorem, which we shall need in the next section. 
Theorem 3.4.3. Let $\{\xi(x), x \geq 0\}$ be a stable Lévy process of index $\alpha \in(1,2]$ with skewness parameter $\beta=-1$. Set

$$
\mu=|\cos (\pi \alpha / 2)|^{-1} .
$$

Then

$$
\lim _{n \rightarrow \infty} \frac{1}{n} \mathbf{E}\left[\exp \left(\sup _{x \in[0, n]}(\xi(x)-\mu x)\right)\right]=(\alpha-1)|\cos (\pi \alpha / 2)|^{-1} .
$$

Proof. We have $\psi(\lambda)=\mu\left(\lambda^{\alpha}-\lambda\right)$. It follows that $\psi^{-1}(0)=1$. Moreover, a simple calculation shows that

$$
\psi^{-1}(\varepsilon)=1+\frac{\varepsilon}{\mu(\alpha-1)}+o(\varepsilon), \quad \varepsilon \rightarrow 0+.
$$

We have, by the previous theorem,

$$
\mathbf{P}\left[\sup _{x \in[0, \tau(q)]}(\xi(x)-\mu x)>p\right]=\exp \left(\psi^{-1}(q) p\right),
$$

where $\tau(q)$ is an exponential stopping time with parameter $q$, independent of $\xi$. With the notation

$$
F_{l}(p)=\mathbf{P}\left[\sup _{x \in[0, l]}(\xi(x)-\mu x) \leq p\right]
$$

we obtain

$$
\int_{0}^{\infty} q \exp (-q l)\left(1-F_{l}(p)\right) d l=\exp \left(\psi^{-1}(q) p\right) .
$$

We need to compute $\lim _{l \rightarrow \infty} f(l) / l$ where

$$
f(l)=\mathbf{E}\left[\exp \left(\sup _{x \in[0, l]}(\xi(x)-\mu x)\right)\right]-1=\int_{0}^{\infty} e^{p}\left(1-F_{l}(p)\right) d p .
$$

To this end, we compute the Laplace transform of $f$ and use Karamata's Tauberian theorem (see e.g. [54, p.192]). First use the integration by parts formula to obtain

$$
\hat{f}(q)=\int_{0}^{\infty} e^{-q l} d f(l)=q \int_{0}^{\infty} f(l) e^{-q l} d l .
$$


Using 3.8 we get

$$
\hat{f}(q)=q \int_{0}^{\infty} \int_{0}^{\infty} e^{-q l} e^{p}\left(1-F_{l}(p)\right) d p d l .
$$

By 3.7 we obtain

$$
\hat{f}(q)=\int_{0}^{\infty} \exp \left(\psi^{-1}(q) p\right) e^{p} d p=\frac{1}{\psi^{-1}(q)-1} .
$$

It follows from $(3.6)$ that

$$
\hat{f}(q) \sim \mu(\alpha-1) q^{-1}, \quad q \rightarrow 0+.
$$

Now use Karamata's Tauberian theorem to obtain

$$
f(l) \sim \mu(\alpha-1) l, \quad l \rightarrow \infty .
$$

This finishes the proof.

Corollary 3.4.4. Let $\left\{\xi_{i}(x), x>0\right\}, i=1, \ldots, d$ be independent stable Lévy processes of index $\alpha \in(1,2)$ with skewness parameter $\beta=-1$. Set

$$
\mu=|\cos (\pi \alpha / 2)|^{-1} .
$$

Define a new process $\left\{Y\left(s_{1}, \ldots, s_{d}\right), s_{1} \geq 0, \ldots, s_{d} \geq 0\right\}$ by

$$
Y\left(s_{1}, \ldots, s_{d}\right)=\xi_{1}\left(s_{1}\right)+\ldots+\xi_{d}\left(s_{d}\right)-\mu\left(s_{1}+\ldots+s_{d}\right) .
$$

For $n \geq 0$ set

$$
G(n)=\mathbf{E}\left[\exp \left(\sup _{0 \leq s_{1}, \ldots, s_{d} \leq n} Y\left(s_{1}, \ldots, s_{d}\right)\right)\right]
$$

Then

$$
\frac{1}{n^{d}} \lim _{n \rightarrow \infty} G(n)=(\alpha-1)^{d}|\cos (\pi \alpha / 2)|^{-d} .
$$

Proof. For $i=1, \ldots, d$ define

$$
G_{i}(n)=\mathbf{E}\left[\exp \left(\sup _{0 \leq s \leq n}\left(\xi_{i}(s)-\mu s\right)\right)\right] .
$$

Then $G(n)=G_{1}(n) \ldots G_{d}(n)$ and it remains to use Theorem 3.4.3. 


\subsection{Proof of Theorem 3.1.1: Local Maxima}

This section is devoted to the proof of the following theorem, describing the asymptotic behavior of the high excursion probability of the random field $X$ over a fixed compact set.

Theorem 3.5.1. Let $K \subset \mathbb{H}$ be compact set of finite positive Jordan measure. Then, as $u \rightarrow \infty$,

$$
\mathbf{P}\left[\sup _{t \in K} X(t)>u\right] \sim C_{\alpha} \int_{K} \frac{d x d y}{y^{2}} u^{2 \alpha /(\alpha-1)}(1-\Phi(u)) .
$$

where

$$
C_{\alpha}=(\alpha-1)^{2}|\cos (\pi \alpha / 2)|^{2 /(\alpha-1)} \alpha^{-2 \alpha /(\alpha-1)} .
$$

Proof. Recall that $q=q(u)$ and $w=w(u)$ were defined in Section 3.3 as

$$
w=w(u)=\frac{\alpha-1}{\alpha B_{\alpha}} u^{-1 /(\alpha-1)}
$$

and

$$
q=q(u)=w^{\alpha}
$$

With this notation, Theorem 3.5.1 is equivalent to

$$
\lim _{u \rightarrow \infty} \frac{q^{2}}{1-\Phi(u)} \mathbf{P}\left[\sup _{t \in K} X(t)>u\right]=D_{\alpha} \int_{K} \frac{d x d y}{y^{2}},
$$

where

$$
D_{\alpha}=(\alpha-1)^{2}|\cos (\pi \alpha / 2)|^{-2} \text {. }
$$

We approximate the set $K$ by finite grids. Let $n \in \mathbb{N}$. Recall that for $t=(x, y) \in \mathbb{H}$ we have defined

$$
Q(t, u, n)=\left\{\left(x-s_{x}, y+s_{x}+s_{y}\right) \in \mathbb{H} \mid 0 \leq s_{x} \leq n q(u), 0 \leq s_{y} \leq n q(u)\right\} .
$$

Let $n q \mathbb{Z}^{2}$ denote the set of points in $\mathbb{R}^{2}$ whose coordinates are integer multiples of $n q$. Define

$$
\begin{aligned}
& K^{*}(u, n)=\left\{t \in n q \mathbb{Z}^{2} \mid Q(t, u, n) \cap K \neq \emptyset\right\} \\
& K_{*}(u, n)=\left\{t \in n q \mathbb{Z}^{2} \mid Q(t, u, n) \subset K\right\} .
\end{aligned}
$$


Each a point $p=(x, y) \in \mathbb{H}$ belongs to some square of the form $Q(\tilde{p}, u, n)$ for some $\tilde{p}=(\tilde{x}, \tilde{y}) \in n q \mathbb{Z}^{2}$. Define

$$
f_{u, n}(p)=\mathbf{P}\left[\sup _{t \in Q(\tilde{p}, u, n)} X(t)>u\right] .
$$

Using an obvious inequality

$$
\mathbf{P}\left[\sup _{t \in K} X(t)>u\right] \leq \sum_{s \in K^{*}(u, n)} \mathbf{P}\left[\sup _{t \in Q(s, u, n)} X(t)>u\right],
$$

we obtain

$$
\mathbf{P}\left[\sup _{t \in K} X(t)>u\right] \leq \frac{1}{(q n)^{2}} \int_{K^{*}(u, n)} f_{u, n}(x, y) d x d y
$$

It follows from Proposition 3.3 .9 that

$$
\lim _{u \rightarrow \infty} \frac{1}{1-\Phi(u)} f_{u, n}(p)=G(n / y) .
$$

It follows

$$
\limsup _{u \rightarrow \infty} \frac{q^{2}}{1-\Phi(u)} \mathbf{P}\left[\sup _{t \in K} X(t)>u\right] \leq \frac{1}{n^{2}} \int_{K^{*}(u, n)} G(n / y) d x d y
$$

Since the last inequality holds for every $n \in \mathbb{N}$, we obtain, by letting $n \rightarrow \infty$,

$$
\limsup _{u \rightarrow \infty} \frac{q^{2}}{1-\Phi(u)} \mathbf{P}\left[\sup _{t \in K} X(t)>u\right] \leq \liminf _{n \rightarrow \infty} \int_{K} \frac{G(n / y)}{(n / y)^{2}} \frac{1}{y^{2}} d x d y .
$$

By Corollary 3.4 .4 we have

$$
\lim _{n \rightarrow \infty} \frac{G(n)}{n^{2}}=D_{\alpha}
$$

It follows that

$$
\limsup _{u \rightarrow \infty} \frac{q^{2}}{1-\Phi(u)} \mathbf{P}\left[\sup _{t \in K} X(t)>u\right] \leq D_{\alpha} \int_{K} \frac{d x d y}{y^{2}},
$$

which proves the upper bound in (3.9). In order the theorem we need to obtain a lower estimate for $\mathbf{P}\left[\sup _{t \in K} X(t)>u\right]$. 
By Bonferroni-Inequality

$$
\mathbf{P}\left[\sup _{t \in K} X(t)>u\right] \geq \sum_{s \in K_{*}(u, n)} \mathbf{P}\left[\sup _{t \in Q(s, u, n)} X(t)>u\right]-\sum_{\substack{s_{1}, s_{2} \in K_{*}(u, n) \\ s_{1} \neq s_{2}}} P\left(s_{1}, s_{2}\right),
$$

where

$$
P\left(s_{1}, s_{2}\right)=P\left(s_{1}, s_{2}, u, n\right)=\mathbf{P}\left[\sup _{t \in Q\left(s_{1}, u, n\right)} X(t)>u \wedge \sup _{t \in Q\left(s_{2}, u, n\right)} X(t)>u\right] .
$$

Since, as we have already shown,

$$
\lim _{u \rightarrow \infty} \frac{q^{2}}{1-\Phi(u)} \sum_{s \in K_{*}(u, n)} \mathbf{P}\left[\sup _{t \in Q(s, u, n)} X(t)>u\right]=D_{\alpha} \int_{K} \frac{d x d y}{y^{2}},
$$

it follows that

$$
\liminf _{u \rightarrow \infty} \frac{q^{2}}{1-\Phi(u)} \mathbf{P}\left[\sup _{t \in K} X(t)>u\right]
$$

can be estimated from below by

$$
D_{\alpha} \int_{K} \frac{d x d y}{y^{2}}-\limsup _{u \rightarrow \infty} \frac{q^{2}}{1-\Phi(u)} \sum_{\substack{s_{1}, s_{2} \in K_{*}(u, n) \\ s_{1} \neq s_{2}}} P\left(s_{1}, s_{2}\right)
$$

Note that this holds for every $n$. Thus, it sufficies to show that

$$
\limsup _{n \rightarrow \infty} \limsup _{u \rightarrow \infty} \frac{q^{2}}{1-\Phi(u)} \sum_{\substack{s_{1}, s_{2} \in K_{*}(u, n) \\ s_{1} \neq s_{2}}} P\left(s_{1}, s_{2}\right)=0
$$

The rest of this section is devoted to the proof of (3.10).

Take sufficiently small $\varepsilon>0$. Then (3.10) would follow from

$$
\limsup _{u \rightarrow \infty} \frac{q^{2}}{1-\Phi(u)} \sum_{\substack{s_{1}, s_{2} \in K_{*}(u, n) \\ d\left(s_{1}, s_{2}\right)>\varepsilon}} P\left(s_{1}, s_{2}\right)=0
$$

and

$$
\limsup _{n \rightarrow \infty} \limsup _{u \rightarrow \infty} \frac{q^{2}}{1-\Phi(u)} \sum_{\substack{s_{1}, s_{2} \in K_{*}(u, n) \\ 0<d\left(s_{1}, s_{2}\right) \leq \varepsilon}} P\left(s_{1}, s_{2}\right)=0
$$


where $d(\cdot, \cdot)$ denotes the euclidian distance in $\mathbb{R}^{2}$.

Before we proceed to the proof of (3.11) and (3.12) we make some remarks. Take $s_{1}=\left(x_{1}, x_{1}+y_{1}\right)$ in $K_{*}(u, n)$. If, say, $s=(x, y) \in Q\left(s_{1}, u, n\right)$ then we may write $x=x_{1}-\delta_{x}, x+y=x_{1}+y_{1}+\delta_{y}$ for some $\delta_{x}, \delta_{y} \in[0, n q]$. The following inequality is evident

$$
X(s)=X(x, y)=\frac{\xi(x+y)-\xi(x)}{y^{1 / \alpha}} \leq \frac{\xi(x+y)-\xi(x)}{y_{1}^{1 / \alpha}} .
$$

It follows that

$$
X(s) \leq \frac{\xi\left(x_{1}+y_{1}\right)-\xi\left(x_{1}\right)}{y_{1}^{1 / \alpha}}+\frac{\xi\left(x_{1}\right)-\xi\left(x_{1}-\delta_{x}\right)}{y_{1}^{1 / \alpha}}+\frac{\xi\left(x_{1}+y_{1}+\delta_{y}\right)-\xi\left(x_{1}+y_{1}\right)}{y_{1}^{1 / \alpha}} .
$$

Now take $s_{1}=\left(x_{1}, x_{1}+y_{1}\right)$ and $s_{2}=\left(x_{2}, x_{2}+y_{2}\right)$. Then $P\left(s_{1}, s_{2}\right)$ is smaller than the probability that both

$$
\sup _{\delta_{x}, \delta_{y} \in[0, n q]} \frac{\xi\left(x_{1}+y_{1}+\delta_{y}\right)-\xi\left(x_{1}-\delta_{x}\right)}{y_{1}^{1 / \alpha}}>u
$$

and

$$
\sup _{\delta_{x}, \delta_{y} \in[0, n q]} \frac{\xi\left(x_{2}+y_{2}+\delta_{y}\right)-\xi\left(x_{2}-\delta_{x}\right)}{y_{2}^{1 / \alpha}}>u .
$$

This probability can be estimated from above by

$\mathbf{P}\left[\sup _{\delta_{x}, \delta_{y} \in[0, n q]} \frac{\xi\left(x_{1}+y_{1}+\delta_{y}\right)-\xi\left(x_{1}-\delta_{x}\right)}{y_{1}^{1 / \alpha}}+\frac{\xi\left(x_{2}+y_{2}+\delta_{y}\right)-\xi\left(x_{2}-\delta_{x}\right)}{y_{2}^{1 / \alpha}}>2 u\right]$.

Thus, we obtain

$$
P\left(s_{1}, s_{2}\right) \leq P_{1}\left(s_{1}, s_{2}\right)+\ldots+P_{5}\left(s_{1}, s_{2}\right)
$$

where

$$
P_{1}=\mathbf{P}\left[\frac{\xi\left(x_{1}+y_{1}\right)-\xi\left(x_{1}\right)}{y_{1}^{1 / \alpha}}+\frac{\xi\left(x_{2}+y_{2}\right)-\xi\left(x_{2}\right)}{y_{2}^{1 / \alpha}}>(2-\eta) u\right]
$$




$$
\begin{aligned}
& P_{2}=\mathbf{P}\left[\sup _{\delta_{x} \in[0, n q]} \frac{\xi\left(x_{1}\right)-\xi\left(x_{1}-\delta_{x}\right)}{\left.y_{1}^{1 / \alpha}>\eta u / 4\right],}\right. \\
& P_{3}=\mathbf{P}\left[\sup _{\delta_{x} \in[0, n q]} \frac{\xi\left(x_{2}\right)-\xi\left(x_{2}-\delta_{x}\right)}{y_{2}^{1 / \alpha}}>\eta u / 4\right], \\
& P_{4}=\mathbf{P}\left[\sup _{\delta_{y} \in[0, n q]} \frac{\xi\left(x_{1}+y_{1}+\delta_{y}\right)-\xi\left(x_{1}+y_{1}\right)}{y_{1}^{1 / \alpha}}>\eta u / 4\right], \\
& P_{5}=\mathbf{P}\left[\sup _{\delta_{y} \in[0, n q]} \frac{\xi\left(x_{2}+y_{2}+\delta_{y}\right)-\xi\left(x_{2}+y_{2}\right)}{y_{1}^{1 / \alpha}}>\eta u / 4\right]
\end{aligned}
$$

and $\eta>0$ is to be chosen later.

In order to estimate $P_{1}$ the following remark will be useful. Denote by $y$ the length of the (possibly empty) interval $\left[x_{1}, x_{1}+y_{1}\right] \cap\left[x_{2}, x_{2}+y_{2}\right]$. Then the random variable

$$
X\left(s_{1}\right)+X\left(s_{2}\right)=\frac{\xi\left(x_{1}+y_{1}\right)-\xi\left(x_{1}\right)}{y_{1}^{1 / \alpha}}+\frac{\xi\left(x_{2}+y_{2}\right)-\xi\left(x_{2}\right)}{y_{2}^{1 / \alpha}}
$$

has the same distribution as

$$
f\left(s_{1}, s_{2}\right) \xi(1),
$$

where where $\xi(1)$ is a standard stable variable of index $\alpha$ with skewness parameter $\beta=-1$ and

$$
f\left(s_{1}, s_{2}\right)=\left(2+\left(\frac{y^{1 / \alpha}}{y_{1}^{1 / \alpha}}+\frac{y^{1 / \alpha}}{y_{2}^{1 / \alpha}}\right)^{\alpha}-\left(\frac{y}{y_{1}}+\frac{y}{y_{2}}\right)\right)^{1 / \alpha} .
$$

If $s_{1}=s_{2}$ then $f\left(s_{1}, s_{2}\right)=2$, it is not difficult to show that otherwise $f\left(s_{1}, s_{2}\right)<2$. Note also that $f$ is continuous on $\mathbb{H} \times \mathbb{H}$.

Proof of (3.11). It sufficies to prove that

$$
\limsup _{u \rightarrow \infty} \frac{q^{2}}{1-\Phi(u)} \sum_{\substack{s_{1}, s_{2} \in K_{*}(u, n) \\ d\left(s_{1}, s_{2}\right)>\varepsilon}} P_{1}\left(s_{1}, s_{2}\right)=0, \quad \forall n \in \mathbb{N}
$$


and

$$
\limsup _{u \rightarrow \infty} \frac{q^{2}}{1-\Phi(u)} \sum_{\substack{s_{1}, s_{2} \in K_{*}(u, n) \\ d\left(s_{1}, s_{2}\right)>\varepsilon}} P_{i}\left(s_{1}, s_{2}\right)=0, \quad \forall n \in \mathbb{N}, i=2,3,4,5 .
$$

We prove 3.21 .

From the continuity of $f$ it follows that $f$ is bounded away from 2 for $d\left(s_{1}, s_{2}\right)>\varepsilon$, i.e. we can find $\delta>0$ such that, say,

$$
f\left(s_{1}, s_{2}\right)<2-3 \delta, \quad d\left(s_{1}, s_{2}\right)>\varepsilon .
$$

We now set $\eta=\delta$ in (3.14) and (3.15)-3.19).

$$
\begin{aligned}
P_{1}\left(s_{1}, s_{2}\right)= & \mathbf{P}\left[\xi(1)>(2-\delta) u / f\left(s_{1}, s_{2}\right)\right] \leq \\
& \mathbf{P}[\xi(1)>(1+\delta) u] \leq \\
& \exp \left(-\left(B_{\alpha}+\delta_{1}\right) u^{\alpha /(\alpha-1)}\right)
\end{aligned}
$$

for some $\delta_{1}>0$. Since the sum in (3.21) contains at most $C /(q n)^{4}$ terms, which grows polynomially as $u \rightarrow \infty$, we have

$$
\frac{q^{2}}{1-\Phi(u)} \sum_{\substack{s_{1}, s_{2} \in K_{*}(u, n) \\ d\left(s_{1} s_{2}\right)>\varepsilon}} P_{1}\left(s_{1}, s_{2}\right) \leq \operatorname{Pol}(u) \frac{1}{1-\Phi(u)} \exp \left(-\left(B_{\alpha}+\delta_{1}\right) u^{\alpha /(\alpha-1)}\right)
$$

which converges to 0 as $u \rightarrow \infty$ by. Here, $\operatorname{Pol}(u)$ stands for $u^{N}$ for some large $N$. This proves 3.21 .

We prove (3.22). It sufficies to consider $P_{2}$, since $P_{3}, P_{4}$ and $P_{5}$ can be treated analogously. Since $y_{1}^{1 / \alpha}$ is bounded away from zero as long as $s_{1} \in K$, we have

$$
\begin{aligned}
P_{2}\left(s_{1}, s_{2}\right) \leq & \mathbf{P}\left[\sup _{\delta_{x} \in[0, n q]} \xi\left(x_{1}\right)-\xi\left(x_{1}-\delta_{x}\right)>C \eta u\right]= \\
& \mathbf{P}\left[\sup _{z \in[0, n q]} \xi(z)>C \eta u\right]=\mathbf{P}\left[\sup _{z \in[0,1]} \xi(z)>C(n q)^{-1 / \alpha} \eta u\right] .
\end{aligned}
$$

Since $C, n$ and $\eta$ are constant and $q^{-1 / \alpha} \rightarrow \infty$ as $u \rightarrow \infty$, we have $C(n q)^{-1 / \alpha} \eta u>$ $2 u$ for all sufficiently large $u$. Using this and Proposition 3.3.3, we obtain, for some $\delta_{1}>0$ and sufficiently large $u$,

$$
P_{2}\left(s_{1}, s_{2}\right) \leq \mathbf{P}\left[\sup _{z \in[0,1]} \xi(z)>2 u\right] \leq \exp \left(-\left(B_{\alpha}+\delta_{1}\right) u^{\alpha /(1-\alpha)}\right) .
$$


Now the number of terms in 3.22 grows polynomially in $u$ and we obtain

$$
\frac{q^{2}}{1-\Phi(u)} \sum_{\substack{s_{1}, s_{2} \in K_{*}(u, n) \\ d\left(s_{1}, s_{2}\right)>\varepsilon}} P_{2}\left(s_{1}, s_{2}\right) \leq \operatorname{Pol}(u) \frac{1}{1-\Phi(u)} \exp \left(-\left(B_{\alpha}+\delta_{1}\right) u^{\alpha /(\alpha-1)}\right),
$$

which converges to 0 as $u \rightarrow \infty$. This proves 3.22 .

Proof of 3.12 . We have to prove that

$$
\limsup _{n \rightarrow \infty} \limsup _{u \rightarrow \infty} \frac{q^{2}}{1-\Phi(u)} \sum_{\substack{s_{1}, s_{2} \in K_{*}(u, n) \\ 0<d\left(s_{1}, s_{2}\right) \leq \varepsilon}} P\left(s_{1}, s_{2}\right)=0
$$

Fix $s_{1}=\left(x_{1}, y_{1}\right)$ and $s_{2}=\left(x_{2}, y_{2}\right)$ in $K_{*}(u, n)$ such that $d\left(s_{1}, s_{2}\right) \leq \varepsilon$. We may write $x_{2}=x_{1}-a n q, x_{2}+y_{2}=x_{1}+y_{1}+b n q$ for some $a, b \in Z$. Suppose for simplicity that $a, b \geq 0$, other cases can be treated analogously. Recall that

$$
P\left(s_{1}, s_{2}\right)=P\left(s_{1}, s_{2}, u, n\right)=\mathbf{P}\left[\sup _{t \in Q\left(s_{1}, u, n\right)} X(t)>u \wedge \sup _{t \in Q\left(s_{2}, u, n\right)} X(t)>u\right] .
$$

Recall also (see (3.13) ) that this probability is smaller than

$\mathbf{P}\left[\sup _{\delta_{x}, \delta_{y} \in[0, n q]} \frac{\xi\left(x_{1}+y_{1}+\delta_{y}\right)-\xi\left(x_{1}-\delta_{x}\right)}{y_{1}^{1 / \alpha}}+\frac{\xi\left(x_{2}+y_{2}+\delta_{y}\right)-\xi\left(x_{2}-\delta_{x}\right)}{y_{2}^{1 / \alpha}}>2 u\right]$.

This is not greater than

$$
\mathbf{P}\left[T+\sup _{\delta_{x}, \delta_{y} \in[0, n q]} \tilde{T}\left(\delta_{x}, \delta_{y}\right)>2 u\right]
$$

where $T=T\left(s_{1}, s_{2}, n, u\right)$ and $\tilde{T}=\tilde{T}\left(s_{1}, s_{2}, n, u, \delta_{x}, \delta_{y}\right)$ are defined by

$$
\begin{gathered}
T=\frac{\xi\left(x_{1}+y_{1}\right)-\xi\left(x_{1}\right)}{y_{1}^{1 / \alpha}}+\frac{\xi\left(x_{1}+y_{1}\right)-\xi\left(x_{1}\right)}{y_{2}^{1 / \alpha}}+\frac{\xi\left(x_{1}-n q\right)-\xi\left(x_{2}\right)}{y_{2}^{1 / \alpha}}+ \\
\frac{\xi\left(x_{2}+y_{2}\right)-\xi\left(x_{1}+y_{1}+n q\right)}{y_{2}^{1 / \alpha}}
\end{gathered}
$$

and

$$
\tilde{T}=T_{1}+T_{2}+T_{3}+T_{1}^{\prime}+T_{2}^{\prime}+T_{3}^{\prime}
$$


where

$$
\begin{array}{ll}
T_{1}=\frac{\xi\left(x_{2}\right)-\xi\left(x_{2}-\delta_{2 x}\right)}{y_{2}^{1 / \alpha}}, & T_{1}^{\prime}=\frac{\xi\left(x_{2}+y_{2}+\delta_{2 x}\right)-\xi\left(x_{2}+y_{2}\right)}{y_{2}^{1 / \alpha}} \\
T_{2}=\frac{\xi\left(x_{1}-\delta_{1 x}\right)-\xi\left(x_{1}-n q\right)}{y_{2}^{1 / \alpha}}, & T_{2}^{\prime}=\frac{\xi\left(x_{1}+y_{1}+n q\right)-\xi\left(x_{1}+y_{1}+\delta_{1 y}\right)}{y_{2}^{1 / \alpha}} \\
T_{3}=\frac{\xi\left(x_{1}\right)-\xi\left(x_{1}-\delta_{1 x}\right)}{y_{1}^{1 / \alpha}}, & T_{3}^{\prime}=\frac{\xi\left(x_{1}+y_{1}+\delta_{1 y}\right)-\xi\left(x_{1}+y_{1}\right)}{y_{1}^{1 / \alpha}}
\end{array}
$$

Note that $T, T_{1}, T_{2}, T_{3}, T_{1}^{\prime}, T_{2}^{\prime}, T_{3}^{\prime}$ are independent. Consequently, $T$ and $\tilde{T}$ are independent. Note also that $T$ has the same distribution as

$$
g\left(s_{1}, s_{2}, n, u\right) \xi(1)
$$

where $\xi(1)$ is standard $\alpha$-stable random variable with skewness parameter $\beta=-1$ and

$$
g\left(s_{1}, s_{2}, n, u\right)=\left(\left(1+\left(\frac{y_{1}}{y_{2}}\right)^{1 / \alpha}\right)^{\alpha}+1-\frac{y_{1}}{y_{2}}-\frac{2 n q}{y_{2}}\right)^{1 / \alpha}
$$

It is not difficult to see that $g\left(s_{1}, s_{2}, n, u\right) \leq 2$. Recall that $x_{2}=x_{1}$ - anq and $x_{2}+y_{2}=x_{1}+y_{1}+$ bnq. Recall also that $d\left(s_{1}, s_{2}\right) \leq \varepsilon$ with $\varepsilon$ sufficiently small. It is easy to deduce that

$$
g\left(s_{1}, s_{2}, n, u\right)>2-c_{1}(a+b) n q
$$

for some constant $c_{1}>0$.

In order to estimate $\tilde{T}$ the following lemma is useful

Lemma 3.5.2. Let $\xi_{1}, \ldots, \xi_{d}, \eta_{1}, \ldots, \eta_{d}$ be independent random variables satisfying

$$
\mathbf{P}\left[\eta_{i}>u\right]<C_{i} \mathbf{P}\left[\xi_{i}>u\right], \quad i=1, \ldots, d
$$

for each $u \in \mathbb{R}$ and some constants $C_{i}>0$. Then

$$
\mathbf{P}\left[\eta_{1}+\ldots+\eta_{d}>u\right]<C_{1} \ldots C_{d} \mathbf{P}\left[\xi_{1}+\ldots+\xi_{d}>u\right]
$$

for each $u \in \mathbb{R}$. 
Proof of Lemma 3.5.2. It sufficies to prove the lemma for $d=2$ (then we can use induction). So let $F_{1}$ (resp. $F_{2}$ ) be the distribution function of $\xi_{1}\left(\right.$ resp. $\left.\xi_{2}\right)$. Let $G_{1}\left(\right.$ resp. $\left.G_{2}\right)$ be the distribution function of $\eta_{1}$ (resp. $\eta_{2}$ ). We have

$$
1-G_{1}(u)<C_{1}\left(1-F_{1}(u)\right) \quad \text { and } \quad 1-G_{2}(u)<C_{2}\left(1-F_{2}(u)\right) \text {. }
$$

It follows

$$
\mathbf{P}\left[\eta_{1}+\eta_{2}>u\right]=\int_{-\infty}^{+\infty}\left(1-G_{1}(u-v)\right) d G_{2}(v)<\int C_{1}\left(1-F_{1}(u)\right) d G_{2}(v) .
$$

Since this integral is equal to $C_{1} \mathbf{P}\left[\xi_{1}+\eta_{2}>u\right]$, we obtain

$$
\mathbf{P}\left[\eta_{1}+\eta_{2}>u\right]<C_{1} \mathbf{P}\left[\xi_{1}+\eta_{2}>u\right]
$$

Now repeat the procedure:

$$
\mathbf{P}\left[\xi_{1}+\eta_{2}>u\right]<\int\left(1-G_{2}(u-v)\right) d F_{1}(v) \leq C_{2} \int\left(1-F_{2}(u-v)\right) d F_{1}(v) .
$$

Since the last integral is equal to $\mathbf{P}\left[\xi_{1}+\xi_{2}>u\right]$, we obtain

$$
\mathbf{P}\left[\xi_{1}+\eta_{2}>u\right]<C_{2} \mathbf{P}\left[\xi_{1}+\xi_{2}>u\right] .
$$

The lemma follows from 3.24 and 3.25 .

Let $\zeta_{1}, \ldots, \zeta_{7}$ be independent standard $\alpha$-stable with $\beta=-1$. Set

$$
\eta_{1}=\sup T_{1}, \eta_{2}=\sup T_{2}, \eta_{3}=\sup T_{3}, \eta_{4}=\sup T_{1}^{\prime}, \eta_{5}=\sup T_{2}^{\prime}, \eta_{6}=\sup T_{3}^{\prime},
$$

where the sup are taken over $\delta_{x}, \delta_{y} \in[0, n q]$. Define $\eta_{7}=T$.

Set furthermore

$$
\xi_{i}=C(n q)^{1 / \alpha} \zeta_{i}, \quad i=1, \ldots, 6
$$

and $\xi_{7}=g\left(s_{1}, s_{2}, n, u\right) \zeta_{7}$ for some big constant $C$. Then it follows from Proposition 3.3.3 that the conditions of Lemma 3.5.2 are satisfied. It follows from the lemma that

$$
\mathbf{P}\left[T+\sup _{\delta_{x}, \delta_{y} \in[0, n q]} \tilde{T}\left(\delta_{x}, \delta_{y}\right)>2 u\right]<C^{\prime} \mathbf{P}\left[\xi_{1}+\ldots+\xi_{7}>2 u\right] .
$$


Now, $\xi_{1}+\ldots+\xi_{7}$ has the same distribution as $\tilde{g}\left(s_{1}, s_{2}, n, u\right) \xi(1)$ where $\xi(1)$ is standard $\alpha$-stable with $\beta=-1$ and

$$
\tilde{g}\left(s_{1}, s_{2}, n, u\right)=\left(g\left(s_{1}, s_{2}, n, u\right)+6 C^{\alpha} n q\right)^{1 / \alpha} .
$$

We obtain

$$
P\left(s_{1}, s_{2}\right) \leq \mathbf{P}\left[T+\sup _{\delta_{x}, \delta_{y} \in[0, n q]} \tilde{T}\left(\delta_{x}, \delta_{y}\right)>2 u\right]<C^{\prime} \mathbf{P}[\xi(1)>(2 / \tilde{g}) u]
$$

It is easy to deduce from 3.23 and the definition of $\tilde{g}$ that for $d\left(s_{1}, s_{2}\right) \leq \varepsilon$ and $\varepsilon$ sufficiently small

$$
\tilde{g}\left(s_{1}, s_{2}, n, u\right)>2-c_{2}(a+b) n q
$$

for some constant $c_{2}>0$. It follows that

$$
2 / \tilde{g}\left(s_{1}, s_{2}, n, u\right)<1+c_{3}(a+b) n q
$$

for some $c_{3}$. We obtain

$$
P\left(s_{1}, s_{2}\right)<C^{\prime} \mathbf{P}\left[\xi(1)>\left(1+c_{3}(a+b) n q\right) u\right]=C^{\prime} \mathbf{P}\left[\xi(1)>u+c_{4}(a+b) n w\right] .
$$

From Proposition 3.3 .3 we conclude that

$$
P\left(s_{1}, s_{2}\right)<(1-\Phi(u)) \exp \left(-c_{4}(a+b) n\right) .
$$

Let $s_{1} \in K_{*}(u, n)$ be fixed. Then

$$
\sum_{\substack{s_{2} \in K_{*}(u, n) \\ 0<d\left(s_{1}, s_{2}\right) \leq \varepsilon}} P_{1}\left(s_{1}, s_{2}\right) \leq C(1-\Phi(u)) \sum_{a, b \in \mathbb{Z}} e^{-c_{4}(|a|+|b|) n} \leq C^{\prime}(1-\Phi(u)) .
$$

Now we sum over $s_{1} \in K_{*}(u, n)$

$$
\sum_{\substack{s_{1}, s_{2} \in K_{*}(u, n) \\ 0<d\left(s_{1}, s_{2}\right) \leq \varepsilon}} P_{1}\left(s_{1}, s_{2}\right) \leq C^{\prime \prime}(q n)^{-2}(1-\Phi(u)) .
$$

It follows that

$$
\limsup _{u \rightarrow \infty} \frac{q^{2}}{1-\Phi(u)} \sum_{\substack{s_{1}, s_{2} \in K_{*}(u, n) \\ 0<d\left(s_{1}, s_{2}\right) \leq \varepsilon}} P_{1}\left(s_{1}, s_{2}\right) \leq C^{\prime \prime} n^{-2}
$$

This finishes the proof of 3.12 and of Theorem 3.5.1. 


\subsection{Proof of Theorem 3.1.1: Global Maxima}

In this section we finish the proof of Theorem 3.1 .3 (recall that it is a rescaled version of Theorem 3.1.1.

Let $\tau \in \mathbb{R}$ be fixed. Let $u=u(n)=a_{n}+b_{n} \tau$ with $a_{n}, b_{n}$ defined by (3.1) and (3.2). Note that $u \rightarrow \infty \Leftrightarrow n \rightarrow \infty$.

Remark 3.6.1. We have, as $u, n \rightarrow \infty$,

$$
C_{\alpha} u^{2 \alpha /(\alpha-1)}(1-\Phi(u)) \sim e^{-\tau} / n
$$

where $C_{\alpha}=(\alpha-1)^{2}|\cos (\pi \alpha / 2)|^{2 /(\alpha-1)} \alpha^{-2 \alpha /(\alpha-1)}$ as in Theorem 3.5.1.

Divide $\mathbb{H}$ into rectangles

$$
R_{k, l}=\left[2^{l+1} k, 2^{l+1}(k+1)\right] \times\left[2^{l}, 2^{l+1}\right], \quad k, l \in \mathbb{Z} .
$$

\section{Remark 3.6.2.}

$$
\int_{R_{k, l}} \frac{d x d y}{y^{2}}=1
$$

The next remark follows from Theorem 3.5.1

\section{Remark 3.6.3.}

$$
\mathbf{P}\left[\sup _{t \in R_{k, l}} X(t)>u\right] \sim \frac{e^{-\tau}}{n} .
$$

Recall that $H(n)$ is the triangle $\{(x, y) \in \mathbb{H} \mid x \in[0, n], y \in[1, n-x]\}$. We need to show that

$$
\lim _{n \rightarrow \infty} \mathbf{P}\left[\sup _{t \in H(n)} X(t)>u\right]=\exp \left(-e^{-\tau}\right) .
$$

As in Section 2.3, it is easy to show that we can replace $H(n)$ by the union of $n+o(n)$ rectangles of the form $R_{k, l}$ which are contained in $H(n)$. Moreover, in order to simplify the notation, we shall assume that the number of rectangles is exactly $n$. So, we replace $H(n)$ by a union of $n$ rectangles of the form $R_{k, l}$. Let $\varepsilon>0$. For each rectangle $R_{k, l}$ consider a smaller rectangle $R_{k, l}(\varepsilon)$ centered at the same point as $R_{k, l}$ and satisfying

$$
\int_{R_{k, l}(\varepsilon)} \frac{d x d y}{y^{2}}=1-\varepsilon .
$$


Set

$$
H(n, \varepsilon)=\bigcup_{R_{k, l} \subset H(n)} R_{k, l}(\varepsilon) .
$$

The next lemma is analogous to Lemma 2.3.5.

Lemma 3.6.4. For some constant $c_{1}>0$

$$
0 \leq \mathbf{P}\left[\sup _{t \in H(n, \varepsilon)} X(t) \leq u\right]-\mathbf{P}\left[\sup _{t \in H(n)} X(t) \leq u\right] \leq c_{1} \varepsilon
$$

We are going to prove the following

\section{Lemma 3.6.5.}

$$
\lim _{n \rightarrow \infty} \mathbf{P}\left[\sup _{t \in H(n, \varepsilon)} X(t) \leq u\right]=e^{-(1-\varepsilon) e^{-\tau}} .
$$

It is easy to see, that Lemma 3.6.5 and Lemma 3.6.4 together imply 3.26) and, consequently, Theorem 3.1 .3 .

The rest of the setion is devoted to the proof of Lemma 3.6.5. First, we introduce some notational simplifications. Take some big but fixed $n$. In order to simplify the notation denote the rectangles of the form $R_{k, l}(\varepsilon)$ which are contained in $H(n, \varepsilon)$ by $R_{1}, \ldots, R_{n}$. We write $R$ for $H(n, \varepsilon)$. Note that $\mathbf{P}\left[\sup _{t \in R_{i}} X(t)>u\right] \sim(1-\varepsilon) e^{-\tau} / n$.

If the events $\left\{\right.$ "sup $\left.\sup _{t \in R_{i}} X(t)>u^{\prime \prime}, i=1, \ldots, n\right\}$ were independent, we would obtain (3.26) immediately by the Poisson Limit Theorem. However, the events are dependent and we need an additional argument which shows that the Poisson Limit Theorem can still be applied. In Section 2.3, we have used Berman's inequality in order to show the approximate independence of events. Unfortunately, Berman's Inequality is not available in the $\alpha$-stable setting, so wee need to use another method.

Let $A$ be the event " $\sup _{t \in R} X(t)>u$ ". For $i=1, \ldots, n$ let $A_{i}$ be the the event " $\sup _{t \in R_{i}} X(t)>u$ ". Then $A=A_{1} \cup \ldots \cup A_{n}$. We are going to estimate $\mathbf{P}[A]$ using the Bonferroni Inequality. To this end, we define, for $k=1, \ldots, n$,

$$
S_{k}=\sum_{1 \leq i_{1}<\ldots<i_{k} \leq n} \mathbf{P}\left[A_{i_{1}} \cap \ldots \cap A_{i_{k}}\right] .
$$


Lemma 3.6.6. For each fixed $k$

$$
\lim _{n \rightarrow \infty} S_{k}=\frac{\left((1-\varepsilon) e^{-\tau}\right)^{k}}{k !}
$$

Proof of Lemma 3.6.6. Let

$$
S_{k}^{\text {indep }}=\sum \mathbf{P}\left[A_{i_{1}} \cap \ldots \cap A_{i_{k}}\right]
$$

where the sum is taken over all independent tuples $1 \leq i_{1}<\ldots<i_{d} \leq n$, i.e. tuples for which $A_{i_{1}}, \ldots, A_{i_{d}}$ are independent. Then it is evident that

$$
S_{k} \geq S_{k}^{\text {indep }}
$$

Now, for each independent tuple we have

$$
\mathbf{P}\left[A_{i_{1}} \cap \ldots \cap A_{i_{k}}\right]=\mathbf{P}\left[A_{1}\right]^{k} \sim\left((1-\varepsilon) e^{-\tau} / n\right)^{k} .
$$

It is not difficult to show that the number of independent tuple is $\sim n^{k} / k$ !. We obtain

$$
\liminf _{n \rightarrow \infty} S_{k}(n) \geq \frac{\left((1-\varepsilon) e^{-\tau}\right)^{k}}{k !} .
$$

Now we need to prove the converse inequality. It sufficies to show that

$$
S_{k}^{d e p}=\sum \mathbf{P}\left[A_{i_{1}} \cap \ldots \cap A_{i_{k}}\right]=o(1), \quad n \rightarrow \infty,
$$

where the sum is taken over all tuples for which (at least two of) the events $A_{i_{1}}, \ldots, A_{i_{k}}$ are dependent.

It is not difficult to show that the number of dependent tuples is $O\left(n^{k-1}\right)$ as $n \rightarrow \infty$. We were done, if we could prove that $\mathbf{P}\left[A_{i_{1}} \cap \ldots \cap A_{i_{k}}\right]=o\left(1 / n^{k-1}\right)$ with uniform $o$ for each dependent tuple. Unfortunately, this is not true since, as we shall see, the probability $\mathbf{P}\left[A_{i_{1}} \cap \ldots \cap A_{i_{k}}\right]$ depends strongly on how the corresponding rectangles $R_{i_{1}}, \ldots, R_{i_{k}}$ are clustered together. Roughly speaking, if the rectangles do not cluster then we have $\mathbf{P}\left[A_{i_{1}} \cap \ldots \cap A_{i_{k}}\right]=$ $O\left(1 / n^{k-\delta}\right)$. Here, $\delta>0$ is some small number depending on the precise notion of clustering. If the rectangles form $1 \leq c<k$ clusters, we have $\mathbf{P}\left[A_{i_{1}} \cap \ldots \cap A_{i_{k}}\right]=O\left(1 / n^{(1+\gamma)(c-\delta)}\right)$. Here, $\gamma>0$ is a small number depending on $\varepsilon$. Now, as we shall see, the number of dependent tuples which do not cluster is $O\left(n^{k-1}\right)$, and the number of tuples which form exactly $c$ clusters, $1 \leq c<k$, is $O\left(n^{c}\right)$. It follows from the above that $S_{k}^{d e p}=o(1)$ as $n \rightarrow \infty$. 
We make the above argument more precise. Recall that each $R_{i}$ has the form $\left[2^{l+1} k, 2^{l+1}(k+1)\right] \times\left[2^{l}, 2^{l+1}\right]$ for some $k, l \in \mathbb{Z}$. Set $J_{i}=\left[2^{l+1} k, 2^{l+1}(k+2)\right]$. We say that two rectangles $R_{i_{1}}$ and $R_{i_{2}}$ are almost disjoint if the corresponding intervals satisfy one of the inequalities

$$
\operatorname{len}\left(J_{i_{1}}\right)>C \operatorname{len}\left(J_{i_{1}} \cap J_{i_{2}}\right) \text { or } \operatorname{len}\left(J_{i_{2}}\right)>C \operatorname{len}\left(J_{i_{1}} \cap J_{i_{2}}\right)
$$

where $C$ is some large constant. Now take some dependent tuple $R_{i_{1}}, \ldots, R_{i_{k}}$. We say that to rectangles from the tuple, say $R_{i_{1}}$ and $R_{i_{2}}$, belong to the same cluster if one can find a finite sequence of rectangles which belong to the tuple starting with $R_{i_{1}}$, ending $R_{i_{2}}$ and such that each two neighboring rectangles from the sequence are not almost disjoint. Now the collection $R_{i_{1}}, \ldots, R_{i_{k}}$ can be represented as a disjoint union of a finite number, say $c \leq k$ clusters. Let

$$
S_{k, c}^{d e p}=\sum \mathbf{P}\left[A_{i_{1}} \cap \ldots \cap A_{i_{k}}\right],
$$

where the sum is taken over all dependent tuples which consist of exactly $c$ clusters. Then

$$
S_{k}^{d e p}=S_{k, k}^{d e p}+\left(S_{k, k-1}^{d e p}+\ldots+S_{k, 1}^{d e p}\right) .
$$

We prove that $S_{k, k}^{d e p}=o(1), n \rightarrow \infty$. Recall that there are at most $O\left(n^{k-1}\right)$ dependent tuples. Thus it sufficies to prove that $\mathbf{P}\left[A_{i_{1}} \cap \ldots \cap A_{i_{k}}\right]=o\left(1 / n^{k-1}\right)$ for each dependent tuple $i_{1}, \ldots, i_{k}$ consisting of $k$ clusters. A rather technical but standard argument, similar to that used in Section 3.3 , shows that $\mathbf{P}\left[A_{i_{1}} \cap\right.$ $\left.\ldots \cap A_{i_{k}}\right]=o\left(1 / n^{k-\delta}\right)$ where $\delta=\delta(C)$ goes to 0 as $C \rightarrow \infty$. It remains to choose $C$ large enough to ensure that $\delta<1$.

We prove that $S_{k, c}^{d e p}=o(1), n \rightarrow \infty$, where $c=1, \ldots, k-1$. The number of tuples which form exactly $c$ clusters is $O\left(n^{c}\right)$. It sufficies to show that $\mathbf{P}\left[A_{i_{1}} \cap \ldots \cap A_{i_{k}}\right]=o\left(1 / n^{c}\right)$ for each tuple consisting of $c \leq k-1$ clusters. Using methods similar to that of Section 3.3 it is possible to show that $\mathbf{P}\left[A_{i_{1}} \cap \ldots \cap A_{i_{k}}\right]=O\left(\left(1 / n^{1+\gamma}\right)^{c-\delta}\right)$ for each tuple consisiting of $c \leq k-1$ clusters. Here, $\delta=\delta(C) \rightarrow 0, C \rightarrow \infty$ and $\gamma=\gamma(\varepsilon) \rightarrow 0, \varepsilon \rightarrow 0$. For a fixed $\varepsilon$ choose $C$ so large that $(1+\gamma)(c-\delta)>c$. We obtain $\mathbf{P}\left[A_{i_{1}} \cap \ldots \cap A_{i_{k}}\right]=$ $o\left(1 / n^{c}\right)$ and , consequently, $S_{k, c}^{\text {dep }}=o(1)$.

This finishes the proof of Lemma 3.6.6.

Proof of Lemma 3.6.5. The Bonferroni inequalities say that, for each $l \in$ $\mathbb{N}$,

$$
\mathbf{P}[A] \geq S_{1}-S_{2}+S_{3}-\ldots-S_{2 l}
$$


and

$$
\mathbf{P}[A] \leq S_{1}-S_{2}+S_{3}-\ldots+S_{2 l+1} .
$$

By the previous lemma, we obtain for each fixed $l \in N$

$$
\liminf _{n \rightarrow \infty} P(A) \geq \sum_{k=1}^{2 l}(-1)^{k-1} \frac{\left((1-\varepsilon) e^{-\tau}\right)^{k}}{k !}
$$

and

$$
\limsup _{n \rightarrow \infty} P(A) \leq \sum_{k=1}^{2 l+1}(-1)^{k-1} \frac{\left((1-\varepsilon) e^{-\tau}\right)^{k}}{k !}
$$

Now let $l \rightarrow \infty$. Lemma 3.6 .5 is proved.

Proof of Theorem 3.1.3. Use Lemmas 3.6.5 and 3.6.4 and let $\varepsilon$ go to 0 . 


\section{Chapter 4}

\section{Almost sure limiting behavior of self-normalized increments}

\subsection{Introduction}

In [52], Shao proved the following conjecture of Révész [50, §14.3]:

Theorem 4.1.1. Let $\left\{X_{i}: i \in \mathbb{N}\right\}$ be independent identically distributed random variables with $\mathbf{P}\left[X_{i}=1\right]=\mathbf{P}\left[X_{i}=-1\right]=1 / 2$. Set $S_{n}=X_{1}+\ldots+$ $X_{n}$. Then, almost surely,

$$
\lim _{n \rightarrow \infty} \frac{1}{\sqrt{2 \log n}} \max _{j, j+k \in\{1, \ldots, n\}} \frac{S_{j+k}-S_{j}}{\sqrt{k}}=1 .
$$

Shao considers also more general distributions. He has proved the following

Theorem 4.1.2. Let $\left\{X_{i}: i \in \mathbb{N}\right\}$ be i.i.d. random variables. Suppose that $\mathbf{E}\left[X_{i}\right]=0, \mathbf{E}\left[X_{i}^{2}\right]=1$ and that $\varphi(t)=\mathbf{E}\left[e^{t X_{1}}\right]$ exists finitely in some interval containing 0. Set $S_{n}=X_{1}+\ldots+X_{n}$. Define $\rho(t)=\inf _{x \geq 0} \varphi(t) \exp (-t x)$ and $\alpha(c)=\sup \{x \geq 0: \rho(x) \geq \exp (-1 / c)\}$. Then, almost surely,

$$
\lim _{n \rightarrow \infty} \frac{1}{\sqrt{2 \log n}} \max _{j, j+k \in\{1, \ldots, n\}} \frac{S_{j+k}-S_{j}}{\sqrt{k}}=\alpha^{*} \in[1, \infty],
$$

where

$$
\alpha^{*}=\sup _{c>0} \frac{\alpha(c)}{\sqrt{2 / c}}
$$


Shao's proof was simplified by Steinebach [53]. He deduces Shao's Theorem from the Erdös-Renyi Law of large numbers (see [24] or [18]) and the following result

Theorem 4.1.3. Let $\left\{X_{i}: i \in \mathbb{N}\right\}$ be i.i.d. random variables. Suppose that $\mathbf{E}\left[X_{i}\right]=0, \mathbf{E}\left[X_{i}^{2}\right]=1$ and that $\varphi(t)=\mathbf{E}\left[e^{t X_{1}}\right]$ exists finitely in some interval containing 0. Set $S_{n}=X_{1}+\ldots+X_{n}$. Define $\rho(t)=\inf _{x \geq 0} \varphi(t) \exp (-t x)$ and $\alpha(c)=\sup \{x \geq 0: \rho(x) \geq \exp (-1 / c)\}$. Then, almost surely,

$$
\lim _{n \rightarrow \infty} \max _{j, j+k \in\{1, \ldots, n\}} \frac{S_{j+k}-S_{j}}{k \alpha(k / \log n)}=1 .
$$

The latter result was extended in [37] to variables with subexponential tails. Our goal is to extend Theorems 4.1 .2 and 4.1 .3 to multidimensional arrays of i.i.d. random variables. Note that both Shao and Steinebach use in their proofs results of Hanson and Russo [28, which are based on Komlos-MajorTusnady strong approximation [35]. Since these results are not available for arrays, we have to use another (and actually more elementary) method. We need only the following classical large deviations theorem, see e.g. [25, Chapter XVI, §7].

Theorem 4.1.4. Let $\left\{X_{i}, i \in \mathbb{N}\right\}$ be i.i.d. random variables satisfying $\mathbf{E}\left[X_{i}\right]=$ $0, \mathbf{E}\left[X_{i}^{2}\right]=1$. Suppose that the moment generating function $\varphi(t)=\mathbf{E}\left[e^{t X_{1}}\right]$ exists in some interval containing 0 . Let $\{x(n), n \in \mathbb{N}\}$ be a sequence satisfying $x(n)=o\left(n^{1 / 6}\right), n \rightarrow \infty$. Then, as $n \rightarrow \infty$,

$$
\mathbf{P}\left[\frac{X_{1}+X_{2}+\ldots+X_{n}}{\sqrt{n}}>x(n)\right] \sim \mathbf{P}[N>x(n)],
$$

where $N \sim N(0,1)$ is a standard normal variable.

Throughout the rest of this chapter let $\left\{X_{\mathbf{n}}, \mathbf{n}=\left(n_{1}, \ldots, n_{d}\right) \in \mathbb{N}^{d}\right\}$ be a $d$-dimensional array of i.i.d. random variables. We denote the typical indices by $\mathbf{n}=\left(n_{1}, \ldots, n_{d}\right), \mathbf{m}=\left(m_{1}, \ldots, m_{d}\right)$ etc. If $m_{i} \leq n_{i}, i=1, \ldots, d$, we write $\mathbf{m} \leq \mathbf{n}$. Define $|\mathbf{n}|=n_{1} \ldots n_{d}$

A set of the form

$$
R(\mathbf{m}, \mathbf{n})=\left\{m_{1}, \ldots, n_{1}\right\} \times \ldots \times\left\{m_{d}, \ldots, n_{d}\right\}, \quad \mathbf{m} \leq \mathbf{n},
$$

is called discrete rectangle. Let $\mathcal{R}$ be the collection of all discrete rectangles. In order to simplify the notation we write $R(\mathbf{n})$ for

$$
R((1, \ldots, 1), \mathbf{n})=\left\{1, \ldots, n_{1}\right\} \times \ldots \times\left\{1, \ldots, n_{d}\right\} .
$$


Let $\mathcal{R}(\mathbf{n})$ be the collection of all discrete rectangles contained in $R(\mathbf{n})$. For a discrete rectangle $R$ define

$$
S_{R}=\sum_{\mathbf{n} \in R} X_{\mathbf{n}}
$$

For a finite set $A$ let $|A|$ denote the number of its elements. Let $\sigma_{R}=\sqrt{|R|}$. Set

$$
M(\mathbf{n})=\max _{R \in \mathcal{R}(\mathbf{n})} \frac{S_{R}}{\sigma_{R}}
$$

Finally, we make some assumptions concerning the distribution of $X_{\mathbf{n}}$. We suppose that $\mathbf{E}\left[X_{\mathbf{n}}\right]=0, \mathbf{E}\left[X_{\mathbf{n}}^{2}\right]=1$ and that the cumulative moment generating function $\varphi(t)=\mathbf{E}\left[e^{t X_{\mathbf{n}}}\right]$ exists finitely in some interval containing 0 . Define as in 53 .

$$
\rho(t)=\inf _{x \geq 0} \varphi(t) \exp (-t x) ; \quad \alpha(c)=\sup \{x \geq 0: \rho(x) \geq \exp (-1 / c)\} .
$$

Set

$$
\alpha^{*}=\sup _{c>0} \frac{\alpha(c)}{\sqrt{2 / c}}
$$

It is easy to prove that $\alpha(c) \sim \sqrt{2 / c}$ as $c \rightarrow+\infty$ and thus $\alpha \geq 1$. As is shown in [52], $\alpha^{*}$ is finite if and only if $\mathbf{E}\left[\exp \left(t X_{\mathbf{n}}^{2}\right)\right]$ exists for some $t>0$. If $X_{\mathbf{n}}$ is normal, Poisson or Bernoulli distributed then $\alpha^{*}=1$.

Our goal is to prove the following theorem which is a higher-dimensional generalization of Theorem 4.1.2.

Theorem 4.1.5. Let $\left\{X_{n}, \boldsymbol{n} \in \mathbb{N}^{d}\right\}$ be a d-dimensional array of i.i.d. random variables. Then, with the above notation and under the above assumptions,

almost surely.

$$
\lim _{\boldsymbol{n} \rightarrow \infty} \frac{1}{\sqrt{2 \log |\boldsymbol{n}|}} M(\boldsymbol{n})=\alpha^{*}
$$

In order to prove the above theorem we need the following analogue of Theorem 4.1.3.

Theorem 4.1.6. Under the assumptions of the previous theorem

$$
\lim _{\boldsymbol{n} \rightarrow \infty} \max _{R \in \mathcal{R}(\boldsymbol{n})} \frac{S_{R}}{|R| \alpha\left(\frac{|R|}{\log |\boldsymbol{n}|}\right)}=1
$$

almost surely. 
The lower bound in this theorem follows from the following generalization of the Erdös-Renyi Law of large numbers

Theorem 4.1.7 (Multidimensional Erdös-Renyi Law). Let $c>0$. Then, almost surely,

$$
\lim _{\boldsymbol{n} \rightarrow \infty} \max _{\substack{R \in \mathcal{R}(\boldsymbol{n}) \\|R| \leq c \log |\boldsymbol{n}|}} \frac{S_{R}}{c \log |\boldsymbol{n}|}=\alpha(c) .
$$

The maximum is taken over all rectangles in $\mathcal{R}(\boldsymbol{n})$ containing less then $[c \log |\boldsymbol{n}|]$ points.

\subsection{Proof of Theorem 4.1.5}

Proof of Theorem 4.1.7. The original proof of Erdös-Renyi applies with minor changes, see [24].

Proof of Theorem 4.1.6. First note that in order to prove the lower bound it is sufficient to take $|R|=[c \log |\mathbf{n}|]$ for $c>0$ and use Theorem 4.1.7, see 53 for details.

We prove the upper bound:

$$
\limsup _{\mathbf{n} \rightarrow \infty} \max _{R \in \mathcal{R}(\mathbf{n})} \frac{S_{R}}{|R| \alpha\left(\frac{|R|}{\log |\mathbf{n}|}\right)} \leq 1 .
$$

We split the set $\mathcal{R}(\mathbf{n})$ into "small" and "big" rectangles. A rectangle is small if it contains not more then $k_{\mathbf{n}}$ points for some multidimensional sequence $\left\{k_{\mathbf{n}}, \mathbf{n} \in \mathbb{N}^{d}\right\}$ to be specified later. We need the following lemma from [39]

Lemma 4.2.1. Let $R$ be a discrete rectangle. Let $\varepsilon>0, m>0$. Then

$$
\mathbf{P}\left[\frac{S_{R}}{|R| \alpha\left(\frac{|R|}{m}\right)}>(1+\varepsilon)\right] \leq \exp (-(1+\varepsilon) m) .
$$

The next lemma (see [53]) estimates the maximum over small rectangles.

Lemma 4.2.2. Let $\left\{k_{\boldsymbol{n}}, \boldsymbol{n} \in \mathbb{N}^{d}\right\}$ be an increasing multidimensional integervalued sequence satisfying $k_{n}=O\left(|\boldsymbol{n}|^{\delta}\right)$ for each $\delta>0$. Then

$$
\limsup _{\boldsymbol{n} \rightarrow \infty} \max _{\substack{R \in \mathcal{R}(\boldsymbol{n}) \\|R| \leq k_{n}}} \frac{S_{R}}{|R| \alpha\left(\frac{|R|}{\log |\boldsymbol{n}|}\right)} \leq 1 .
$$


Proof. Use Lemma 4.2.1, see [53] for details.

Now we are going to treat the maximum over big rectangles.

Lemma 4.2.3. Let $\left\{k_{\boldsymbol{n}}, \boldsymbol{n} \in \mathbb{N}^{d}\right\}$ be an increasing multidimensional integervalued sequence such that $k_{\boldsymbol{n}} /(\log |\boldsymbol{n}|)^{3 d} \rightarrow \infty$ as $\boldsymbol{n} \rightarrow \infty$. Then

$$
\limsup _{n \rightarrow \infty} \max _{\substack{R \in \mathcal{R}(n) \\|R|>k_{n}}} \frac{S_{R}}{|R| \alpha\left(\frac{|R|}{\log |\boldsymbol{n}|}\right)} \leq 1 .
$$

Proof. In the one-dimensional case, Steinebach 53] deduces this lemma from the results of Hanson-Russo [28]. Since the Komlos-Major-Tusnady strong approximation theorem [35], which is crucial for [28], is not available for arrays, we have to use another method.

First recall from [53] that $\alpha(c) \sim \sqrt{2 / c}, c \rightarrow \infty$. It follows that

$$
|R| \alpha\left(\frac{|R|}{\log |\mathbf{n}|}\right) \sim \sqrt{|R|} \sqrt{2 \log |\mathbf{n}|} .
$$

So we have to prove that

$$
\limsup _{\mathbf{n} \rightarrow \infty} \frac{1}{\sqrt{2 \log \mathbf{n}}} \max _{\substack{R \in \mathcal{R}(\mathbf{n}) \\|R|>k_{\mathbf{n}}}} \frac{S_{R}}{\sqrt{|R|}} \leq 1 .
$$

This follows via the Borel-Cantelli lemma from the following

Proposition 4.2.4. Write $\mathcal{R}^{*}(\boldsymbol{n})$ for the collection of all rectangles of the form $R(\boldsymbol{m}, \boldsymbol{n}), \boldsymbol{m} \leq \boldsymbol{n}$. Then, for each $\varepsilon>0$, there exists $\delta=\delta(\varepsilon)>0$ such that for all sufficiently large $\boldsymbol{n} \in \mathbb{N}^{d}$

$$
\mathbf{P}\left[\frac{1}{\sqrt{2 \log |\boldsymbol{n}|}} \max _{\substack{R \in \mathcal{R}^{*}(\boldsymbol{n}) \\|R|>k_{n}}} \frac{S_{R}}{\sqrt{|R|}}>1+\varepsilon\right]<\frac{1}{|\boldsymbol{n}|^{1+\delta}} .
$$

Proof of the proposition. Take $\sigma>1$ so close to 1 that $(\sigma-1)^{-d / 2} \frac{\varepsilon}{2}>2$. We may suppose that $\sigma^{k} \notin \mathbb{N}, k \in \mathbb{N}$. Let

$$
K(\mathbf{n})=K(\mathbf{n}, \sigma)=\left\{\mathbf{k}=\left(k_{1}, \ldots, k_{d}\right) \quad \mid \quad k_{i} \in \mathbb{N}, \sigma^{k_{i}}<n_{i}, i=1, \ldots, d\right\} .
$$

For $\mathbf{k} \in K(\mathbf{n})$ define $Q(\mathbf{k})=Q\left(k_{1}, \ldots, k_{d}\right)$ and $S(\mathbf{k})=S\left(k_{1}, \ldots, k_{d}\right)$ by

$$
Q(\mathbf{k})=\left\{\left[n_{1}-\sigma^{k_{1}}\right], \ldots, n_{1}\right\} \times \ldots \times\left\{\left[n_{d}-\sigma^{k_{d}}\right], \ldots, n_{d}\right\}
$$


and

$$
S(\mathbf{k})=\left\{\left[n_{1}-\sigma^{k_{1}+1}\right], \ldots,\left[n_{1}-\sigma^{k_{1}}\right]\right\} \times \ldots \times\left\{\left[n_{d}-\sigma^{k_{d}+1}\right], \ldots,\left[n_{d}-\sigma^{k_{d}}\right]\right\} .
$$

For each $\mathbf{m}=\left(m_{1}, \ldots, m_{d}\right) \leq \mathbf{n}$ one can find $\mathbf{k}=\mathbf{k}(\mathbf{m})=\left(k_{1}, \ldots, k_{d}\right)$ with $\mathbf{m} \in S\left(k_{1}, \ldots, k_{d}\right)$. Recall that

$$
R(\mathbf{m}, \mathbf{n})=\left\{m_{1}, \ldots, n_{1}\right\} \times \ldots \times\left\{m_{d}, \ldots, n_{d}\right\}, \quad \mathbf{m} \leq \mathbf{n} .
$$

Define

$$
P(\mathbf{m})=P(\mathbf{m}, \mathbf{n}, \sigma)=R(\mathbf{m}, \mathbf{n}) \backslash Q(\mathbf{k}(\mathbf{m})) .
$$

Then $R(\mathbf{m}, \mathbf{n})$ is a disjoint union of $Q(\mathbf{k}(\mathbf{m}))$ and $P(\mathbf{m})$ and it follows that

$$
\frac{S_{R(\mathbf{m}, \mathbf{n})}}{\sqrt{|R(\mathbf{m}, \mathbf{n})|}} \leq \frac{S_{Q(\mathbf{k}(\mathbf{m}))}}{\sqrt{|Q(\mathbf{k}(\mathbf{m}))|}}+\frac{S_{P(\mathbf{m})}}{\sqrt{|Q(\mathbf{k}(\mathbf{m}))|}}
$$

Thus

$$
\max _{\substack{\mathbf{m} \leq \mathbf{n} \\|R(\mathbf{m}, \mathbf{n})|>k_{\mathbf{n}}}} \frac{S_{R(\mathbf{m}, \mathbf{n})}}{\sqrt{|R(\mathbf{m}, \mathbf{n})|}} \leq \max _{\substack{\mathbf{k} \in K(\mathbf{n}) \\|Q(\mathbf{k})|>k_{\mathbf{n}}}} \frac{S_{Q(\mathbf{k})}}{\sqrt{|Q(\mathbf{k})|}}+\max _{\substack{\mathbf{m} \leq \mathbf{n} \\|R(\mathbf{m}, \mathbf{n})|>k_{\mathbf{n}}}} \frac{S_{P(\mathbf{m})}}{\sqrt{|Q(\mathbf{k}(\mathbf{m}))|}}
$$

It sufficies to prove that, for some $\delta>0$,

$$
\mathbf{P}\left[\frac{1}{\sqrt{2 \log |\mathbf{n}|}} \max _{\substack{\mathbf{k} \in K(\mathbf{n}) \\|Q(\mathbf{k})|>k_{\mathbf{n}}}} \frac{S_{Q(\mathbf{k})}}{\sqrt{|Q(\mathbf{k})|}}>1+\frac{\varepsilon}{2}\right]<\frac{1}{|\mathbf{n}|^{1+\delta}}
$$

and

$$
\mathbf{P}\left[\frac{1}{\sqrt{2 \log |\mathbf{n}|}} \max _{\substack{\mathbf{m} \leq \mathbf{n} \\|R(\mathbf{m}, \mathbf{n})|>k_{\mathbf{n}}}} \frac{S_{P(\mathbf{m})}}{\sqrt{|Q(\mathbf{k}(\mathbf{m}))|}}>\frac{\varepsilon}{2}\right]<\frac{1}{|\mathbf{n}|^{1+\delta}} .
$$

In order to prove 4.1 note that

$$
\mathbf{P}\left[\frac{1}{\sqrt{2 \log |\mathbf{n}|}} \max _{\substack{\mathbf{k} \in K(\mathbf{n}) \\|Q(\mathbf{k})|>k_{\mathbf{n}}}} \frac{S_{Q(\mathbf{k})}}{\sqrt{|Q(\mathbf{k})|}}>1+\frac{\varepsilon}{2}\right]
$$

is not greater than

$$
\sum_{\substack{\mathbf{k} \in K(\mathbf{n}) \\|Q(\mathbf{k})|>k_{\mathbf{n}}}} \mathbf{P}\left[\frac{S_{Q(\mathbf{k})}}{\sqrt{|Q(\mathbf{k})|}}>\left(1+\frac{\varepsilon}{2}\right) \sqrt{2 \log |\mathbf{n}|}\right]
$$


and use Theorem 4.1.4.

To prove (4.2) note that

$$
\frac{S_{P(\mathbf{m})}}{\sqrt{|Q(\mathbf{k}(\mathbf{m}))|}}=\frac{S_{P(\mathbf{m})}}{\sqrt{|P(\mathbf{m})|}} \frac{\sqrt{|P(\mathbf{m})|}}{\sqrt{|Q(\mathbf{k}(\mathbf{m}))|}} \leq(\sigma-1)^{d / 2} \frac{S_{P(\mathbf{m})}}{\sqrt{|P(\mathbf{m})|}} .
$$

It is evident that

$$
\mathbf{P}\left[\frac{1}{\sqrt{2 \log |\mathbf{n}|}} \max _{\substack{\mathbf{m} \leq \mathbf{n} \\|R(\mathbf{m}, \mathbf{n})|>k_{\mathbf{n}}}} \frac{S_{P(\mathbf{m})}}{\sqrt{|Q(\mathbf{k}(\mathbf{m}))|}}>\frac{\varepsilon}{2}\right]
$$

is not greater than

$$
\sum_{\substack{\mathbf{m} \leq \mathbf{n} \\ R(\mathbf{m}, \mathbf{n}) \mid>k_{\mathbf{n}}}} \mathbf{P}\left[\frac{S_{P(\mathbf{m})}}{\sqrt{|Q(\mathbf{k}(\mathbf{m}))|}}>\frac{\varepsilon}{2} \sqrt{2 \log |\mathbf{n}|}\right] .
$$

Each summand in the last sum is not greater than

$$
\mathbf{P}\left[\frac{S_{P(\mathbf{m})}}{\sqrt{\mid S_{P(\mathbf{m}) \mid}}}>(\sigma-1)^{-d / 2} \frac{\varepsilon}{2} \sqrt{2 \log |\mathbf{n}|}\right]<\mathbf{P}\left[\frac{S_{P(\mathbf{m})}}{\sqrt{\left|S_{P(\mathbf{m})}\right|}}>2 \sqrt{2 \log |\mathbf{n}|}\right],
$$

which is $\sim \frac{1}{|\mathbf{n}|^{4}}$ by Theorem 4.1.4. Note that the use of Theorem 4.1 .4 is justified by the following

Lemma 4.2.5. Suppose that $d \geq 2$. Let $\boldsymbol{m} \leq \boldsymbol{n}$ be such that $|R(\boldsymbol{m}, \boldsymbol{n})|>k_{\boldsymbol{n}}$.

Then

$$
\sqrt{\log |\boldsymbol{n}|}=o\left(|P(\boldsymbol{m})|^{1 / 6} \mid\right), \quad \boldsymbol{n} \rightarrow \infty .
$$

Proof of Lemma 4.2.5. We have to prove that

$$
\lim _{|\mathbf{n}| \rightarrow \infty} \frac{|P(\mathbf{m})|}{(\log |\mathbf{n}|)^{3}}=\infty \text {. }
$$

Recall that $\sigma^{k} \notin \mathbb{N}, k \in \mathbb{N}$. It follows

$$
\begin{gathered}
|P(\mathbf{m})| \geq \prod_{i=1}^{d}\left(\sigma^{k_{i}(\mathbf{m})}+1\right)-\prod_{i=1}^{d} \sigma^{k_{i}(\mathbf{m})} \geq \sum_{i=1}^{d} \sigma^{k_{i}(\mathbf{m})} \geq \sigma^{-1} \sum_{i=1}^{d}\left(n_{i}-m_{i}\right) \geq \\
\sigma^{-1} d\left(\prod_{i=1}^{d}\left(n_{i}-m_{i}\right)\right)^{1 / d}=\sigma^{-1} d|R(\mathbf{m}, \mathbf{n})|^{1 / d} \geq \sigma^{-1} d\left(k_{\mathbf{n}}\right)^{1 / d} .
\end{gathered}
$$

Recall that $k_{\mathbf{n}} / \log |\mathbf{n}|^{3 d} \rightarrow \infty$. Lemma 4.2 .5 is proved. 
It follows

$$
\mathbf{P}\left[\frac{1}{\sqrt{2 \log |\mathbf{n}|}} \max _{\substack{\mathbf{m} \leq \mathbf{n} \\|R(\mathbf{m}, \mathbf{n})|>k_{\mathbf{n}}}} \frac{S_{P(\mathbf{m})}}{\sqrt{|Q(\mathbf{k}(\mathbf{m}))|}}>\frac{\varepsilon}{2}\right]<\frac{1}{|\mathbf{n}|^{3}}<\frac{1}{|\mathbf{n}|^{1+\delta}} .
$$

This finishes the proof of 4.2 and Proposition 4.2.4.

In order to prove Lemma 4.2.3, use Proposition 4.2.4 and the Borel-Cantelli lemma.

The upper bound in Theorem 4.1.6 follows now from Lemmas 4.2.2 and 4.2.3 by choosing $k_{\mathbf{n}}=[\log |\mathbf{n}|]^{r}$ for sufficiently large $r$.

This finishes the proof of Theorem 4.1.6.

Proof of Theorem 4.1.5. The method of [53] can be used.

$$
\liminf _{\mathbf{n} \rightarrow \infty} \frac{1}{\sqrt{2 \log |\mathbf{n}|}} \max _{R \in \mathcal{R}(\mathbf{n})} \frac{S_{R}}{\sqrt{|R|}} \geq \liminf _{\mathbf{n} \rightarrow \infty} \sqrt{\frac{c}{2}} \max _{\substack{R \in \mathcal{R}(\mathbf{n}) \\|R|=[c \log |\mathbf{n}|]}} \frac{S_{R}}{|R|},
$$

which is equal to $\sqrt{\frac{c}{2}} \alpha(c) \leq \alpha^{*}$ by the Erdös-Renyi Law (see Theorem 4.1.7). We prove the upper bound. Note that, by the definition of $\alpha^{*}$,

$$
|R| \alpha\left(\frac{|R|}{\log |\mathbf{n}|}\right) \leq|R| \alpha^{*} \sqrt{\frac{2 \log |\mathbf{n}|}{|R|}}=\alpha^{*} \sqrt{2 \log |\mathbf{n}|} \sqrt{|R|} .
$$

Thus

$$
\begin{aligned}
\frac{1}{\alpha^{*}} \limsup _{\mathbf{n} \rightarrow \infty} \frac{1}{\sqrt{2 \log |\mathbf{n}|}} \max _{R \in \mathcal{R}(\mathbf{n})} \frac{S_{R}}{\sqrt{|R|}}= & \limsup _{\mathbf{n} \rightarrow \infty} \max _{R \in \mathcal{R}(\mathbf{n})} \frac{1}{\alpha^{*}} \frac{1}{\sqrt{2 \log |\mathbf{n}|}} \frac{S_{R}}{\sqrt{|R|}} \leq \\
& \limsup _{\mathbf{n} \rightarrow \infty} \max _{R \in \mathcal{R}(\mathbf{n})} \frac{S_{R}}{|R| \alpha\left(\frac{|R|}{\log |\mathbf{n}|}\right)},
\end{aligned}
$$

which is equal to 1 by Theorem 4.1.6.

This finishes the proof of Theorem 4.1.5. 


\section{Bibliography}

[1] Albin, J.M.P. (1990). On extremal theory for stationary processes. Ann. Probab. 18 92-128.

[2] Albin, J.M.P. (1993). Extremes of totally skewed stable motion. Stat. Probab. Lett. 16 219-224.

[3] Albin, J.M.P. (1999). Extremes of totally skewed $\alpha$-stable processes. Stochastic Processes Appl. 79 185-212.

[4] Albin, J.M.P. On Extremes of Infinitely Divisible OrnsteinUhlenbeck Processes. Available at http://www.math.chalmers.se/ palbin/manuscripts.html

[5] Albin, J.M.P. and Bengtsson,M. On the Asymptotic Behaviour of Lévy processes. Part I: Subexponential and Exponential Processes. Part II: Superexponential Processes. Available at http://www.math.chalmers.se/ palbin/manuscripts.html

[6] Aldous, D.(1989): Probability approximations via the Poisson clumping heuristic. Applied Mathematical Sciences, 77 Springer-Verlag.

[7] Antonini, R. and Weber, M. (2004). The intersective ASCLT. Stochastic Anal. Appl. 22 1009-1025.

[8] Berg, Chr., Christensen, J.P.R. and Ressel, P. (1984). Harmonic analysis on semigroups. Theory of positive definite and related functions. Graduate Texts in Mathematics, 100 New York etc.: Springer-Verlag.

[9] Berkes, I. and Csáki, E. (2001). A universal result in almost sure central limit theory. Stochastic Processes Appl. 94 105-134 
[10] Berkes, I. and Weber, M. (2006). Almost sure versions of the Darling-Erdös theorem. Stat. Probab. Lett. 76 280-290.

[11] Berman, S.M. (1992). Sojourns and extremes of stochastic processes. Wadsworth \& Brooks/Cole Statistics/Probability Series.

[12] Bertoin, J. (1998). Darling-Erdös theorems for normalized sums of i.i.d. variables close to a stable law. Ann. Probab. 26 832-852.

[13] Bertoin, J. (1998). Lévy processes. Cambridge Tracts in Mathematics. 121 Cambridge Univ. Press.

[14] Bickel, P. and Rosenblatt, M. (1973). Two-dimensional random fields. In: Multivar. Analysis III. Proc. 3rd internat. Symp., Dayton 1972, 3-15.

[15] Bingham, N.H. (1973). Maxima of sums of random variables and suprema of stable processes. Z. Wahrscheinlichkeitstheor. Verw. Geb. 26 273-296.

[16] Chan, H. P. and LaI, T. L. (2006). Maxima of asymptotically Gaussian random fields and moderate deviation approximations to boundary crossing probabilities of sums of random variables with multidimensional indices. Ann. Probab. 34 80-121.

[17] Chung, K.L., Erdös, P. and Sirao, T. (1959). On the Lipschitz's condition for Brownian motion. J. Math. Soc. Japan 11 263-274.

[18] Csörgö, M. and RÉVÉsz, P. (1981). Strong approximations in probability and statistics. Probability and Mathematical Statistics. New York-San Francisco-London: Academic Press.

[19] Darling, D.A. and Erdös, P. (1956). A limit theorem for the maximum of normalized sums of independent random variables. Duke Math. J. 23 143-155.

[20] DümbGen, L. and Spokoiny, V.G. (2001). Multiscale testing of qualitative hypotheses. Ann. Stat. 29 124-152.

[21] Dümbgen, L. and Walther,G. (2006). Multiscale inference about a density. Preprint (Extended version: Technical report 56 Univ. of Bern) 
[22] Einmahl, U. (1989). The Darling-Erdös theorem for sums of i.i.d. random variables. Probab. Theory Relat. Fields 82 241-257.

[23] Einmahl, U. and Mason, D.M. (1989). Darling-Erdös theorems for martingales. J. Theor. Probab. 2 437-460.

[24] Erdös, P. and Renyi, A. (1970). On a new law of large numbers. $J$. Anal. Math. 23 103-111.

[25] Feller, W. (1971). An introduction to probability theory and its applications. Vol II. 2nd ed. Wiley Series in Probability and Mathematical Statistics. New York etc.: John Wiley and Sons, Inc.

[26] Fristedt, B. (1964). The behavior of increasing stable processes for both small and large times. J. Math. Mech. 13 849-856.

[27] Gneiting, T. and Schlather, M. (2004). Stochastic models that separate fractal dimension and the Hurst effect. SIAM Rev. 46 269282.

[28] Hanson, D.L. and Russo, R.P. (1983). Some results on increments of the Wiener process with applications to lag sums of i.i.d. random variables. Ann. Probab. 11 609-623.

[29] Hawkes, J. (1971). A lower Lipschitz condition for the stable subordinator. Z. Wahrscheinlichkeitstheor. Verw. Geb. 17 23-32.

[30] Horváth, L. and Shao, Q.-M. (1996). Darling-Erdös-type theorems for sums of Gaussian variables with long-range dependence. Stochastic Processes Appl. 63 117-137.

[31] HüsLER, J. (1990). Extreme values and high boundary crossings of locally stationary Gaussian processes. Ann. Probab. 18 1141-1158.

[32] Ibragimov, I.A. and Linnik, Yu.V. (1971). Independent and stationary sequences of random variables. Groningen: Wolters-Noordhoff Publishing Company.

[33] Ito, K. and McKean, H.P. (1974). Diffusion processes and their sample paths. 2nd pr. Die Grundlehren der mathematischen Wissenschaften. 125 Springer-Verlag. 
[34] Khoshnevisan, D., Levin, D. and Shi, Zh.(2005). Extreme-Value Analysis of the LIL for Brownian Motion Electr. Comm. Probab. 10 Paper 20, pp. 196-206.

[35] Komlos, J., Major, P. and Tusnády, G. (1975). An approximation of partial sums of independent RV's, and the sample DF. I. Z. Wahrscheinlichkeitstheor. Verw. Geb. 32 111-131.

[36] Leadbetter, M.R., Lindgren, G. and Rootzen, H. (1983). Extremes and related properties of random sequences and processes. Springer Series in Statistics. New York - Heidelberg - Berlin: SpringerVerlag.

[37] Lanzinger, H. and Stadtmüller, U. (2000). Maxima of increments of partial sums for certain subexponential distributions. Stochastic Processes Appl. 86 307-322.

[38] LÉvy, P. (1937). Théorie de l'addition des variables aléatoires. Paris: Gauthier-Villars.

[39] Mason, D.M. (1989). An extended version of the Erdös-Rényi strong law of large numbers. Ann. Probab. 17 257-265.

[40] McKean, H.P. (1969). Stochastic integrals. New York-London: Academic Press.

[41] MijnheER, J.L. (1975). Sample path properties of stable processes. Mathematical Centre Tracts. 59 Amsterdam: Mathematisch Centrum.

[42] Mikhaleva, T.L. and Piterbarg, V.I. (1996). On the distribution of the maximum of a Gaussian field with a constant variance on a smooth manifold. Theory Probab. Appl. 41 367-379; translation from Teor. Veroyatn. Primen. 41 438-451.

[43] Pickands, J. (1969). Upcrossing probabilities for stationary Gaussian processes. Trans. Am. Math. Soc. 145 51-73.

[44] Pickands, J. (1969). Asymptotic properties of the maximum in a stationary Gaussian process. Trans. Am. Math. Soc. 145 75-86. 
[45] Piterbarg, V.I. and Fatalov, V.R. (1995). The Laplace method for probability measures in Banach spaces. Russ. Math. Surv. 50 11511239; translation from Usp. Mat. Nauk 50 57-150.

[46] Piterbarg, V.I. (1996). Asymptotic methods in the theory of Gaussian processes and fields. Translations of Mathematical Monographs. 148 Providence, RI: AMS.

[47] Port, S.C. (1989). Stable processes with drift on the line. Trans. Am. Math. Soc. 313 805-841.

[48] Qualls, C. and Watanabe, H. (1972). Asymptotic properties of Gaussian processes. Ann. Math. Stat. 43 580-596.

[49] Qualls, C. and Watanabe, H. (1973). Asymptotic properties of Gaussian random fields. Trans. Am. Math. Soc. 177 155-171.

[50] RÉvÉsz, P. (2005). Random walk in random and non-random environments. 2nd ed. World Scientific.

[51] Samorodnitsky, G. and Taqqu, M.S. (1994). Stable non-Gaussian random processes: stochastic models with infinite variance. Stochastic Modeling. New York, NY: Chapman \& Hall.

[52] Shao, Q.-M. (1995). On a conjecture of Révész. Proc. Am. Math. Soc. 123 575-582.

[53] Steinebach, J. (1997). On a conjecture of Révész and its analogue for renewal processes. In: Szyszkowicz, Barbara (ed.), Asymptotic methods in probability and statistics. A volume in honour of Miklós Csörgö. ICAMPS '97, an international conference at Carleton Univ., Ottawa, Ontario, Canada. Amsterdam: North-Holland/ Elsevier.

[54] Widder, D. V. (1946). The Laplace transform. New York. Princeton Press.

[55] Zolotarev, V.M. (1986). One-dimensional stable distributions. Translations of Mathematical Monographs 65. Providence, R.I.: AMS. 


\section{Lebenslauf}

\begin{tabular}{|c|c|}
\hline Name & Zakhar Kabluchko \\
\hline Geburtsdatum & 13. August 1982 \\
\hline Geburtsort & Bryansk (Russland) \\
\hline Familienstand & ledig \\
\hline $1989-1998$ & Grundschule und Gymnasium 17, Vinniza \\
\hline $1998-2001$ & Studium an der Universität Kiew \\
\hline $2001-2004$ & Studium an der Georg-August-Universität Göttingen \\
\hline $2004-2007$ & $\begin{array}{l}\text { Doktorand am Institut für Mathematische Stochastik, } \\
\text { Göttingen }\end{array}$ \\
\hline seit 01.09.2006 & $\begin{array}{l}\text { Wissenschaftlicher Mitarbeiter am Institut für Mathe- } \\
\text { matische Stochastik }\end{array}$ \\
\hline
\end{tabular}

